\title{
Clifford Analysis with Indefinite Signature
}

\author{
Matvei Libine*and Ely Sandine ${ }^{\dagger}$
}

November 18, 2020

\begin{abstract}
We extend constructions of classical Clifford analysis to the case of indefinite nondegenerate quadratic forms. We define $(p, q)$-left- and right-monogenic functions by means of Dirac operators that factor a certain wave operator. We prove two different versions of Cauchy's integral formulas for these functions. The two formulas arise from dealing with singularities in distinct ways, and are inspired by the methods of [L, FL]. These results indicate the merit of these methods for dealing with singularities.
\end{abstract}

\section{Introduction}

Many results of complex analysis have analogues in quaternionic analysis. In particular, there are analogues of complex holomorphic functions called (left- and right-) regular functions. Cauchy's integral formula for complex holomorphic functions $f(z)$

$$
f\left(z_{0}\right)=\frac{1}{2 \pi i} \oint \frac{f(z)}{z-z_{0}} d z
$$

extends to the quaternionic setting, and its analogues for (left- and right-) regular functions are usually referred to as Cauchy-Fueter formulas. For modern introductions to quaternionic analysis see, for example, [S, CSSS].

Complex numbers $\mathbb{C}$ and quaternions $\mathbb{H}$ are special cases of Clifford algebras. (For an elementary introduction to Clifford algebras see, for example, G].) There is a further extension of complex and quaternionic analysis called Clifford analysis. For Clifford algebras associated to positive definite quadratic forms on real vector spaces, Clifford analysis is very similar to complex and quaternionic analysis (see, for example, [BDS, DSS, GM] and references therein). Furthermore, J. Ryan has initiated the study of Clifford analysis in the setting of complex Clifford algebras [R1, R2].

If a Clifford algebra is associated to a quadratic form that is not positive definite, the function that should serve as the reproducing kernel in the Cauchy type formula has singularities that always intersect the contour of integration, thus rendering a potential reproducing integral formula meaningless. For this reason, most developments of real Clifford analysis

${ }^{*}$ Department of Mathematics, Indiana University, Rawles Hall, 831 East 3rd St, Bloomington, IN 47405

${ }^{\dagger}$ Undergraduate student at Cornell University, Ithaca, NY 14850 
so far have been in the setting of Clifford algebras associated to positive definite quadratic forms.

We develop Clifford analysis in the setting of Clifford algebras associated to indefinite non-degenerate quadratic forms by treating the relevant Clifford algebras as real subalgebras of complex Clifford algebras and, in a certain sense, restricting the complex Dirac operators and functions to the appropriate real subspaces. This is done in complete parallel with K. Imaeda's approach in [I], where he first extends classical quaternionic analysis to the algebra of biquaternions $\mathbb{H}_{\mathbb{C}}=\mathbb{H} \otimes_{\mathbb{R}} \mathbb{C}$ and then "restricts" to the Minkowski space $\mathbb{M}$ by realizing $\mathbb{M}$ as a real form of $\mathbb{H}_{\mathbb{C}}$.

Similarly, the works [L, FL] extend classical quaternionic analysis to split quaternions $\mathbb{H}_{\mathbb{R}}$ (also known as coquaternions). The algebra $\mathbb{H}_{\mathbb{R}}$ is isomorphic to the algebra of real $2 \times 2$ matrices and is another example of a Clifford algebra, this time associated to an indefinite non-degenerate quadratic form on $\mathbb{R}^{2}$. The issue of singularities intersecting the contour of integration is resolved in two different ways, leading to two types of integral formulas. The first method uses biquaternions $\mathbb{H}_{\mathbb{C}}$, which contain the split quaternions $\mathbb{H}_{\mathbb{R}}$ as a real subalgebra. By considering the holomorphic extension of a regular function from $\mathbb{H}_{\mathbb{R}}$ into $\mathbb{H}_{\mathbb{C}}$ and deforming the contour of integration so it no longer intersects the singularities, one obtains the first version of Cauchy's formulas for regular functions in $\mathbb{H}_{\mathbb{R}}$. The second method involves inserting a purely imaginary term $i \varepsilon\left\|X-X_{0}\right\|^{2}$ into the denominator of the reproducing kernel to prevent singularities, then showing that the limit as $\varepsilon \rightarrow 0$ exists and produces another version of Cauchy's formulas for regular functions in $\mathbb{H}_{\mathbb{R}}$.

Methods for dealing with non-positive definite signature setting developed in $[\mathrm{L}, \mathrm{FL}]$ were used in $[\mathrm{RCW}]$ and $[\mathrm{P}]$. Thus $[\mathrm{RCW}]$ extends the results of classical Clifford analysis to the setting of what the authors call split-quaternionic Hermitian Clifford analysis. And [P] extends analysis over octonions to split octonions. Both papers have to deal with exactly the same issue of the set of singularities intersecting the contour of integration, and this issue is resolved in the same way as in $[\mathrm{L}, \mathrm{FL}$. In this paper we use methods of $[\mathrm{L}, \mathrm{FL}]$ to extend constructions of classical Clifford analysis to the case of indefinite non-degenerate quadratic forms. In a larger context, our results suggest that the methods of handling singularities developed in [L, FL] are quite general.

We begin by recalling in Section 2 the construction of the universal Clifford algebras. These constructions are done in both the real and complex cases, and we regard the real Clifford algebra as a subalgebra of the complex Clifford algebra. Following this, in Section 3 we define Dirac operators and introduce left- and right-monogenic functions as functions annihilated by Dirac operators. Both real and complex cases are considered. The complex case was mostly developed by J. Ryan [R1, R2], and the real case is, in a certain sense, the restriction of the complex operators and functions to the real subspace. In Section 4 we introduce Clifford-valued differential forms $D_{n} z, D_{p, q} x$ and establish some of their properties. These forms appear in the statements of Cauchy's formulas for monogenic functions. We also state the classical Cauchy's integral formulas for left- and right-monogenic functions in the positive definite case (Theorem [16). In Section 5 we state and prove the first version of Cauchy's integral formulas for left- and right-monogenic functions (Theorem 20). This version requires having holomorphic extension of the monogenic functions to the complex space and utilizes a deformation of the contour of integration to avoid the set of singularities. In Section [6] we prove the second version of Cauchy's integral formulas for left- and right- 
monogenic functions (Theorem 23). This version involves inserting a purely imaginary term $i \varepsilon\left\|X-X_{0}\right\|^{2}$ into the denominator of the reproducing kernel to prevent singularities, then taking limit as $\varepsilon \rightarrow 0^{+}$. We first establish Theorem 23 up to a constant coefficient (26). Then we pin down the value of the coefficient using the first version of integral formulas (Theorem 201), which is already established (Lemma 33).

This research was made possible by the Indiana University, Bloomington, Math REU (research experiences for undergraduates) program, funded by NSF Award \#1757857. We would like to thank Prof. Chris Connell for organizing and facilitating this wonderful program. We would also like to thank Ms. Mandie McCarthy for her administrative work, the various professors who gave talks, and the other REU students for their company.

\section{Clifford Algebras}

In this section we recall basics of Clifford Algebras and establish notations. For an elementary introduction to Clifford algebras see, for example, G].

Let $V$ be an $n$-dimensional real vector space, and $Q$ a quadratic form on $V$. The form $Q$ uniquely extends to a bilinear form on $V$. Diagonalizing $Q$, we have that there exist integers $p, q$ and orthogonal basis $\left\{e_{1}, \ldots, e_{n}\right\}$ of $(V, Q)$, such that

$$
Q\left(e_{j}\right)= \begin{cases}1 & 1 \leq j \leq p \\ -1 & p+1 \leq j \leq p+q \\ 0 & p+q<j \leq n .\end{cases}
$$

By Sylvester's Law of Inertia, we the numbers $p$ and $q$ are independent of basis chosen. The ordered pair $(p, q)$ is the signature of $(V, Q)$. From this point on we restrict our attention to non-degenerate quadratic forms $Q$, in which case $p+q=n$. If $Q$ is such a form with $q=0$ or $p=0$, then it is positive or negative definite quadratic form respectively.

Recall the standard construction of the universal Clifford algebra associated to $(V, Q)$ as a quotient of the tensor algebra. We start with the tensor algebra over $V$,

$$
\bigotimes V=\mathbb{R} \oplus V \oplus(V \otimes V) \oplus(V \otimes V \otimes V) \oplus \ldots,
$$

consider a set of elements of the tensor algebra

$$
S=\{v \otimes v+Q(v): v \in V\} \quad \subset \bigotimes V,
$$

and let $(S)$ denote the ideal of $\otimes V$ generated by the elements of $S$. Then the universal Clifford algebra associated to $(V, Q)$ can be defined as a quotient

$$
\mathcal{A}_{Q}=\bigotimes V /(S)
$$

We note the sign convention chosen for elements of the ideal is not standard in the literature, resulting in a possible interchange of $p$ and $q$. Let $e_{0} \in \mathcal{A}_{Q}$ denote the multiplicative identity of the algebra. If $v_{1}$ and $v_{2}$ are orthogonal elements of $(V, Q)$,

$$
\begin{aligned}
v_{1} \otimes v_{2}+v_{2} \otimes v_{1}=\left(v_{1}+v_{2}\right) \otimes\left(v_{1}+v_{2}\right)-v_{1} \otimes & v_{1}-v_{2} \otimes v_{2} \\
& =-Q\left(v_{1}+v_{2}\right)+Q\left(v_{1}\right)+Q\left(v_{2}\right)=0 .
\end{aligned}
$$


Thus $\mathcal{A}_{Q}$ is a finite-dimensional algebra over $\mathbb{R}$ generated by $e_{1}, \ldots, e_{n}$, and let $\mathcal{A}_{p, q}$ denote the algebra $\mathcal{A}_{Q}$ constructed from $(V, Q)$, where $Q$ has signature $(p, q)$. We fix an orthogonal basis

$$
\left\{e_{1}, e_{2}, \ldots, e_{p}, \tilde{e}_{p+1}, \ldots, \tilde{e}_{p+q}\right\}
$$

of $V$ such that $Q\left(e_{j}\right)=1$ and $Q\left(\tilde{e}_{j}\right)=-1$ for all applicable $j$. In the positive definite case the basis of $V$ is given by $\left\{e_{1}, e_{2}, \ldots, e_{n}\right\}$, and we let $\mathcal{A}_{n}=\mathcal{A}_{n, 0}$. We have the following relations for $1 \leq i, j \leq n$ with $i \neq j$,

$$
\begin{aligned}
& e_{0}^{2}=e_{0}, \quad e_{i}^{2}=-e_{0}, \quad \tilde{e}_{j}^{2}=e_{0}, \quad e_{0} e_{i}=e_{i} e_{0}=e_{i}, \quad e_{0} \tilde{e}_{i}=\tilde{e}_{i} e_{0}=\tilde{e}_{i}, \\
& e_{i} e_{j}=-e_{j} e_{i}, \quad e_{i} \tilde{e}_{j}=-\tilde{e}_{j} e_{i}, \quad \tilde{e}_{i} \tilde{e}_{j}=-\tilde{e}_{j} \tilde{e}_{i} .
\end{aligned}
$$

We consider subsets $B \subseteq\{1, \ldots, n\}$. If $B$ has $k>0$ elements,

$$
B=\left\{i_{1}, i_{2}, \ldots, i_{k}\right\} \subseteq\{1, \ldots, n\}
$$

with $i_{1}<i_{1}<\cdots<i_{k}$, define

$$
e_{B}=e_{i_{1} i_{2} \ldots i_{k}}=e_{i_{1}} \otimes e_{i_{2}} \otimes \cdots \otimes \tilde{e}_{i_{k}}
$$

or, more precisely, $e_{B}$ is the image of this tensor product in $\mathcal{A}_{Q}$. If $B$ is empty, we set $e_{\emptyset}$ be the identity element $e_{0}$. These elements

$$
\left\{e_{B}: B \subseteq\{1,2, \ldots, n\}\right\}
$$

form a vector space basis of $\mathcal{A}_{p, q}$ over $\mathbb{R}$. We will be especially concerned with the space $\mathbb{R} \oplus V$, which we identify with the vector subspace $\mathbb{R}^{p+q+1} \subset \mathcal{A}_{p, q}$ spanned by

$$
\left\{e_{0}, e_{1}, e_{2}, \ldots, e_{p}, \tilde{e}_{p+1}, \ldots, \tilde{e}_{p+q}\right\} .
$$

Any $X \in \mathbb{R}^{p+q+1}$ can be written as

$$
X=\sum_{j=0}^{p} x_{j} e_{j}+\sum_{j=p+1}^{p+q} \tilde{x}_{j} \tilde{e}_{j} .
$$

In particular, in the positive definite case any $X \in \mathbb{R}^{n+1} \subset \mathcal{A}_{n}$ can be expressed as $X=$ $\sum_{j=0}^{n} x_{j} e_{j}$.

We can also construct a complex universal Clifford algebra from a quadratic space over $\mathbb{C}$. Let $V^{\mathbb{C}}$ be an $n$-dimensional complex vector space with quadratic form $Q$. Diagonalizing $Q$, we have that there exists an orthogonal basis $\left\{e_{1}, \ldots, e_{n}\right\}$ of $V^{\mathbb{C}}$ and integer $p$ such that $Q\left(e_{j}\right)=1$ if $1 \leq j \leq p$ and $Q\left(e_{j}\right)=0$ otherwise. The form $Q$ is non-degenerate if and only if $p=n$, and we only consider this case. We perform a similar construction to (11): form the tensor algebra over $V^{\mathbb{C}}$

$$
\bigotimes V^{\mathbb{C}}=\mathbb{C} \oplus V^{\mathbb{C}} \oplus\left(V^{\mathbb{C}} \otimes V^{\mathbb{C}}\right) \oplus\left(V^{\mathbb{C}} \otimes V^{\mathbb{C}} \otimes V^{\mathbb{C}}\right) \oplus \ldots,
$$

consider a set

$$
S^{\mathbb{C}}=\left\{v \otimes v+Q(v): v \in V^{\mathbb{C}}\right\} \quad \subset \bigotimes V^{\mathbb{C}},
$$


and let $\left(S^{\mathbb{C}}\right)$ denote the ideal generated by $S^{\mathbb{C}}$. Then the complex universal Clifford algebra associated to $\left(V^{\mathbb{C}}, Q\right)$ is a quotient

$$
\mathcal{A}_{Q}^{\mathbb{C}}=\bigotimes V^{\mathbb{C}} /\left(S^{\mathbb{C}}\right)
$$

We also use notation $\mathcal{A}_{n}^{\mathbb{C}}$ for this algebra. The analogues of (2) and (3)-(44) remain valid for $\mathcal{A}_{n}^{\mathbb{C}}$, and the products $e_{B}$ defined as in (5) form a basis of $\mathcal{A}_{n}^{\mathbb{C}}$.

We can also consider $\mathcal{A}_{n}^{\mathbb{C}}$ as a real algebra generated by

$$
e_{0}, e_{1}, e_{2}, \ldots, e_{n}, i e_{0}, i e_{1}, i e_{2}, \ldots, i e_{n}
$$

By the universality property of $\mathcal{A}_{p, q}$, the natural inclusion $\iota: V \hookrightarrow V^{\mathbb{C}}$ defined on basis vectors by

$$
\iota\left(e_{j}\right)=e_{j}, \quad 1 \leq j \leq p, \quad \iota\left(\tilde{e}_{j}\right)=i e_{j}, \quad p+1 \leq j \leq p+q,
$$

extends to an injective $\mathbb{R}$-algebra homomorphism $\iota: \mathcal{A}_{p, q} \hookrightarrow \mathcal{A}_{p+q}^{\mathbb{C}}$. Thus, we can consider $\mathcal{A}_{p, q}$ as a unital real subalgebra of $\mathcal{A}_{p+q}^{\mathbb{C}}$.

We identify the $\mathbb{C}$-span of $e_{0}, e_{1}, \ldots, e_{n}$ with $\mathbb{C}^{n+1} \subset \mathcal{A}_{n}^{\mathbb{C}}$. On $\mathbb{C}^{n+1}$ we have the Clifford conjugation defined by

$$
Z=z_{0} e_{0}+\sum_{j=1}^{n} z_{j} e_{j} \quad \mapsto \quad Z^{+}=z_{0} e_{0}-\sum_{j=1}^{n} z_{j} e_{j}
$$

and the complex conjugation defined by

$$
Z=z_{0} e_{0}+\sum_{j=1}^{n} z_{j} e_{j} \quad \mapsto \quad \bar{Z}=\overline{z_{0}} e_{0}+\sum_{j=1}^{n} \bar{z}_{j} e_{j}
$$

(These conjugations can be extended to all of $\mathcal{A}_{p+q}^{\mathbb{C}}$, but for the purposes of this paper we do not need this.) The Clifford conjugation fixes the $\mathbb{C}$-span of $e_{0}$ identified with $\mathbb{C}$, and the complex conjugation fixes the $\mathbb{R}$-span of $e_{0}, \ldots, e_{n}$ identified with $\mathbb{R}^{n+1}$, and these conjugations can be viewed as reflecting over the respective subspaces.

These two conjugations commute and lead to two useful quadratic forms on $\mathbb{C}^{n+1} \subset \mathcal{A}_{n}^{\mathbb{C}}$. The first such form is

$$
N(Z)=Z Z^{+}=Z^{+} Z=\sum_{j=0}^{n} z_{j}^{2}
$$

(note that it is complex valued). The corresponding bilinear form is

$$
\langle Z, W\rangle=\frac{1}{2}\left(Z^{+} W+Z W^{+}\right)=\sum_{j=0}^{n} z_{j} w_{j} .
$$

We denote by $\mathcal{N}_{n}^{\mathbb{C}}$ the null cone of $N(Z)$ :

$$
\mathcal{N}_{n}^{\mathbb{C}}=\left\{Z \in \mathbb{C}^{n+1} ; N(Z)=0\right\}
$$


then all $Z \in \mathbb{C}^{n+1} \backslash \mathcal{N}_{n}^{\mathbb{C}}$ are invertible with inverse given by $Z^{-1}=N(Z)^{-1} Z^{+}$. The other quadratic form is real valued:

$$
\|Z\|^{2}=\frac{1}{2}\left(Z \bar{Z}^{+}+\bar{Z} Z^{+}\right)=\frac{1}{2}\left(\bar{Z}^{+} Z+Z^{+} \bar{Z}\right)=\sum_{j=0}^{n}\left|z_{j}\right|^{2} .
$$

As was mentioned earlier, we consider $\mathcal{A}_{p, q}$ as a real subalgebra of $\mathcal{A}_{p+q}^{\mathbb{C}}$. We describe the restrictions of these two conjugations and two quadratic forms to $\mathbb{R}^{p+q+1} \subset \mathcal{A}_{p, q} \subset \mathcal{A}_{p+q}^{\mathbb{C}}$ :

$$
\begin{gathered}
X=x_{0} e_{0}+\sum_{j=1}^{p} x_{j} e_{j}+\sum_{j=p+1}^{p+q} \tilde{x}_{j} \tilde{e}_{j} \mapsto X^{+}=x_{0} e_{0}-\sum_{j=1}^{p} x_{j} e_{j}-\sum_{j=p+1}^{p+q} \tilde{x}_{j} \tilde{e}_{j}, \\
X=x_{0} e_{0}+\sum_{j=1}^{p} x_{j} e_{j}+\sum_{j=p+1}^{p+q} \tilde{x}_{j} \tilde{e}_{j} \mapsto \quad \bar{X}=x_{0} e_{0}+\sum_{j=1}^{p} x_{j} e_{j}-\sum_{j=p+1}^{p+q} \tilde{x}_{j} \tilde{e}_{j}, \\
N(X)=X X^{+}=X^{+} X=\sum_{j=0}^{p} x_{j}^{2}-\sum_{j=p+1}^{p+q} \tilde{x}_{j}^{2}, \\
\|X\|^{2}=\frac{1}{2}\left(X \bar{X}^{+}+\bar{X} X^{+}\right)=\frac{1}{2}\left(\bar{X}^{+} X+X^{+} \bar{X}\right)=\sum_{j=0}^{p} x_{j}^{2}+\sum_{j=p+1}^{q} \tilde{x}_{j}^{2} .
\end{gathered}
$$

The bilinear form on $\mathbb{R}^{p+q+1}$ corresponding to $N(X)$ is

$$
\langle X, Y\rangle=\frac{1}{2}\left(X^{+} Y+X Y^{+}\right)=\sum_{j=0}^{p} x_{j} y_{j}-\sum_{j=p+1}^{p+q} \tilde{x}_{j} \tilde{y}_{j} .
$$

In line with the complex case, we consider the null cone

$$
\mathcal{N}_{p, q}=\left\{X \in \mathbb{R}^{p+q+1} ; N(X)=0\right\}
$$

then all $X \in \mathbb{R}^{p+q+1} \backslash \mathcal{N}_{p, q}$ are invertible with inverse given by $X^{-1}=N(X)^{-1} X^{+}$. Finally, we note that in the positive definite case $\mathcal{N}_{n, 0}=\{0\}$.

\section{Dirac Operators, Monogenic Functions and Green's Functions}

In this section we recall the definitions of Dirac operators, monogenic functions and Green's functions in the setting of complex Clifford algebras that were originally introduced in [R1]. Then, using the inclusion $\mathbb{R}^{p+q+1} \subset \mathcal{A}_{p, q} \subset \mathcal{A}_{p+q}^{\mathbb{C}}$, we restrict these notions to define their analogues in the setting of real Clifford algebras $\mathcal{A}_{p, q}$. When we apply these restricted Dirac operators to the restricted Green's functions, we obtain special monogenic functions that will serve as reproducing kernels of Cauchy's integral formulas in the $\mathcal{A}_{p, q}$ setting. 
We introduce linear differential operators on $\mathbb{C}^{n+1}$

$$
\nabla_{\mathbb{C}}^{+}=e_{0} \frac{\partial}{\partial z_{0}}+\sum_{j=1}^{n} e_{j} \frac{\partial}{\partial z_{j}} \quad \text { and } \quad \nabla_{\mathbb{C}}=e_{0} \frac{\partial}{\partial z_{0}}-\sum_{j=1}^{n} e_{j} \frac{\partial}{\partial z_{j}}
$$

which may be applied to functions on the left and on the right. Let $\square_{\mathbb{C}}$ be the complex Laplacian

$$
\square_{\mathbb{C}}=\sum_{j=0}^{n} \frac{\partial^{2}}{\partial z_{j}^{2}}
$$

Then

$$
\nabla_{\mathbb{C}} \nabla_{\mathbb{C}}^{+} f=\nabla_{\mathbb{C}}^{+} \nabla_{\mathbb{C}} f=\square_{\mathbb{C}} f \quad \text { and } \quad g \nabla_{\mathbb{C}} \nabla_{\mathbb{C}}^{+}=g \nabla_{\mathbb{C}}^{+} \nabla_{\mathbb{C}}=\square_{\mathbb{C}} g,
$$

where $f$ is a holomorphic function on $\mathbb{C}^{n+1}$ with values in $\mathcal{A}_{n}^{\mathbb{C}}$ or a left $\mathcal{A}_{n}^{\mathbb{C}}$-module, and $g$ is a holomorphic function on $\mathbb{C}^{n+1}$ with values in $\mathcal{A}_{n}^{\mathbb{C}}$ or a right $\mathcal{A}_{n}^{\mathbb{C}}$-module.

Definition 1. Let $U \subseteq \mathbb{C}^{n+1}$ be an open set, and $M_{n}^{\mathbb{C}}$ a left $\mathcal{A}_{n}^{\mathbb{C}}$-module. A holomorphic function $f: U \rightarrow M_{n}^{\mathbb{C}}$ is called complex left-monogenic if

$$
\nabla_{\mathbb{C}}^{+} f=e_{0} \frac{\partial f}{\partial z_{0}}+\sum_{j=1}^{n} e_{j} \frac{\partial f}{\partial z_{j}}=0
$$

at all points in $U$.

Similarly, let $\tilde{M}_{n}^{\mathbb{C}}$ be a right $\mathcal{A}_{n}^{\mathbb{C}}$-module, then a holomorphic function $g: U \rightarrow \tilde{M}_{n}^{\mathbb{C}}$ is called complex right-monogenic if

$$
g \nabla_{\mathbb{C}}^{+}=\frac{\partial g}{\partial z_{0}} e_{0}+\sum_{j=1}^{n} \frac{\partial g}{\partial z_{j}} e_{j}=0
$$

at all points in $U$.

The factorization (10) leads to a natural method of constructing complex left- and rightmonogenic functions. If $\varphi: U \rightarrow M_{n}^{\mathbb{C}}$ is complex harmonic (i.e., $\square_{\mathbb{C}} \varphi=0$ ), with $U$ and $M_{n}^{\mathbb{C}}$ as in the definition, then $\nabla_{\mathbb{C}} f$ is complex left-monogenic. Similarly, if $\tilde{\varphi}: U \rightarrow \tilde{M}_{n}^{\mathbb{C}}$ is complex harmonic, $\tilde{\varphi} \nabla_{\mathbb{C}}$ is complex right-monogenic.

We restrict these definitions to the subalgebra $\mathcal{A}_{p, q} \subset \mathcal{A}_{p+q}^{\mathbb{C}}$. Thus we introduce linear differential operators

$$
\begin{aligned}
& \nabla_{p, q}^{+}=e_{0} \frac{\partial}{\partial x_{0}}+\sum_{j=1}^{p} e_{j} \frac{\partial}{\partial x_{j}}-\sum_{j=p+1}^{p+q} \tilde{e}_{j} \frac{\partial}{\partial \tilde{x}_{j}} \quad \text { and } \\
& \nabla_{p, q}=e_{0} \frac{\partial}{\partial x_{0}}-\sum_{j=1}^{p} e_{j} \frac{\partial}{\partial x_{j}}+\sum_{j=p+1}^{p+q} \tilde{e}_{j} \frac{\partial}{\partial \tilde{x}_{j}}
\end{aligned}
$$

which may be applied to functions on the left and on the right. Let $\square_{p, q}$ be a wave operator on $\mathbb{R}^{p+q+1}$,

$$
\square_{p, q}=\sum_{j=0}^{p} \frac{\partial^{2}}{\partial x_{j}^{2}}-\sum_{j=p+1}^{p+q} \frac{\partial^{2}}{\partial \tilde{x}_{j}^{2}}
$$


Then

$$
\nabla_{p, q} \nabla_{p, q}^{+} f=\nabla_{p, q}^{+} \nabla_{p, q} f=\square_{p, q} f \quad \text { and } \quad g \nabla_{p, q} \nabla_{p, q}^{+}=g \nabla_{p, q}^{+} \nabla_{p, q}=\square_{p, q} g,
$$

where $f$ is a $\mathcal{C}^{2}$ function on $\mathbb{R}^{p+q+1}$ with values in $\mathcal{A}_{p, q}$ or a left $\mathcal{A}_{p, q}$-module, and $g$ is a $\mathcal{C}^{2}$ function on $\mathbb{R}^{p+q+1}$ with values in $\mathcal{A}_{p, q}$ or a right $\mathcal{A}_{p, q}$-module.

Definition 2. Let $U \subset \mathbb{R}^{p+q+1}$ be an open set, and $M_{p, q}$ a left $\mathcal{A}_{p, q}$-module. $A \mathcal{C}^{1}$ function $f: U \rightarrow M_{p, q}$ is called $(p, q)$-left-monogenic if

$$
\nabla_{p, q}^{+} f=e_{0} \frac{\partial f}{\partial x_{0}}+\sum_{j=1}^{p} e_{j} \frac{\partial f}{\partial x_{j}}-\sum_{j=p+1}^{p+q} \tilde{e}_{j} \frac{\partial f}{\partial \tilde{x}_{j}}=0
$$

at all points in $U$.

Similarly, let $\tilde{M}_{p, q}$ be a right $\mathcal{A}_{p, q}$-module, then a $\mathcal{C}^{1}$ function $g: U \rightarrow \tilde{M}_{p, q}$ is called $(p, q)$-right-monogenic if

$$
g \nabla_{p, q}^{+}=\frac{\partial g}{\partial x_{0}} e_{0}+\sum_{j=1}^{p} \frac{\partial g}{\partial x_{j}} e_{j}-\sum_{j=p+1}^{p+q} \frac{\partial g}{\partial \tilde{x}_{j}} \tilde{e}_{j}=0
$$

at all points in $U$.

The factorization (11) leads to a natural method of constructing $(p, q)$-left and rightmonogenic functions. If $\varphi: U \rightarrow M_{p, q}$ satisfies $\square_{p, q} \varphi=0$, with $U$ and $M_{p, q}$ as in the definition, then $\nabla_{p, q} f$ is $(p, q)$-left-monogenic. Similarly, if $\tilde{\varphi}: U \rightarrow \tilde{M}_{p, q}$ satisfies $\square_{p, q} \tilde{\varphi}=0$, $\tilde{\varphi} \nabla_{p, q}$ is $(p, q)$-right-monogenic.

The following case is an important example of such a construction, in which we consider a Green's function on $\mathbb{C}^{p+q+1} \subset \mathcal{A}_{p+q}^{C}$ and a suitable restriction to $\mathbb{R}^{p+q+1} \subset \mathcal{A}_{p, q}$.

Definition 3. For odd $n \geq 2$, we define the following function $H_{n}(Z): \mathbb{C}^{n+1} \backslash \mathcal{N}_{n}^{\mathbb{C}} \rightarrow \mathbb{C}$ by

$$
H_{n}(Z)=\frac{1}{N(Z)^{\frac{n-1}{2}}}=\frac{1}{\left(\sum_{j=0}^{n} z_{j}^{2}\right)^{\frac{n-1}{2}}} .
$$

We note that if $n$ is even, this may not be well defined. In this case, we introduce a domain

$$
\mathbb{C}_{G}^{n+1}=\mathbb{C}^{n+1} \backslash\left\{Z \in \mathbb{C}^{n+1} ; N(Z) \in \mathbb{R}, N(Z) \leq 0\right\}
$$

and choose a branch of $N(Z)^{\frac{1}{2}}: \mathbb{C}_{G}^{n+1} \rightarrow \mathbb{C}$ with values in the right half-plane $\{w \in \mathbb{C} ; \operatorname{Re} w>$ $0\}$. Then $H_{n}(Z)=\left(N(Z)^{\frac{1}{2}}\right)^{1-n}$ is a well defined function $\mathbb{C}_{G}^{n+1} \rightarrow \mathbb{C}$. From now on we restrict the domain of $H_{n}(Z)$ to $\mathbb{C}_{G}^{n+1}$ regardless of the parity of $n$, as the same proofs hold in either case. Observe that on this domain $H_{n}(Z)$ is complex harmonic, and, since $H_{n}(Z)$ is scalar-valued, $\nabla_{\mathbb{C}} H_{n}(Z)=H_{n}(Z) \nabla_{\mathbb{C}}$ is complex left- and right-monogenic. Thus, we obtain a function on $\mathbb{C}_{G}^{n+1}$ with values in $\mathcal{A}_{n}^{\mathbb{C}}$ that is complex left- and right-monogenic:

Lemma 4. An $\mathcal{A}_{n}^{\mathbb{C}}$-valued function $G_{n}(Z)$ defined on $\mathbb{C}_{G}^{n+1}$ as

$$
G_{n}(Z)=\frac{1}{1-n} \nabla_{\mathbb{C}} H_{n}(Z)=\frac{z_{0}-\sum_{j=1}^{n} z_{j} e_{j}}{\left(\sum_{j=0}^{n} z_{j}^{2}\right)^{\frac{n+1}{2}}}=\frac{Z^{+}}{N(Z)^{\frac{n+1}{2}}}
$$

is complex left- and right-monogenic. 
Restricting this function to $\mathbb{R}^{p+q+1} \subset \mathcal{A}_{p, q}$ as was done with Dirac operators earlier, we obtain the following solution to the wave equation $\square_{p, q} \varphi=0$ and the corresponding $(p, q)$-left- and $(p, q)$-right-monogenic functions. Let

$$
\mathbb{R}_{G}^{p+q+1}=\mathbb{R}^{p+q+1} \backslash\left\{X \in \mathbb{R}^{n+1} ; N(X) \leq 0\right\}=\mathbb{R}^{p+q+1} \cap \mathbb{C}_{G}^{p+q+1} .
$$

Definition 5. For all $p+q \geq 2$, define the functions $H_{p, q}(X): \mathbb{R}_{G}^{p+q+1} \rightarrow \mathbb{R}$ and $G_{p, q}(X)$ : $\mathbb{R}_{G}^{p+q+1} \rightarrow \mathcal{A}_{p, q}$ by

$$
\begin{gathered}
H_{p, q}(X)=\left.H_{p+q}(X)\right|_{\mathbb{R}_{G}^{p+q+1}}=\frac{1}{N(X)^{\frac{p+q-1}{2}}}=\frac{1}{\left(\sum_{j=0}^{p} x_{j}^{2}-\sum_{j=p}^{p+q} \tilde{x}_{j}^{2}\right)^{\frac{p+q-1}{2}}}, \\
G_{p, q}(X)=\left.G_{p+q}(X)\right|_{\mathbb{R}_{G}^{p+q+1}}=\frac{1}{1-p-q} \nabla_{p, q} H_{p, q}(X) \\
=\frac{x_{0}-\sum_{j=1}^{p} x_{j} e_{j}-\sum_{j=p+1}^{p+q} \tilde{x}_{j} \tilde{e}_{j}}{\left(\sum_{j=0}^{p} x_{j}^{2}-\sum_{j=p+1}^{p+q} \tilde{x}_{j}^{2}\right)^{\frac{p+q+1}{2}}}=\frac{X^{+}}{N(X)^{\frac{p+q+1}{2}} .}
\end{gathered}
$$

Consequently, $\square_{p, q} H_{p, q}(X)=0$ and $G_{p, q}$ is $(p, q)$-left- and right-monogenic.

\section{Differential Forms}

In this section we introduce the Clifford-valued differential $n$-forms $D_{n} z$ and $D_{p, q} x$ that will appear in the statement of Cauchy's integral formulas. We also prove several properties of these forms. At the end of the section we state the classical Cauchy's integral formulas for monogenic functions. The complex case originally was developed in [R1].

Definition 6. Let $d V_{\mathbb{C}}$ be the $n+1$ complex holomorphic form on $\mathbb{C}^{n+1}$ :

$$
d V_{\mathbb{C}}=d z_{0} \wedge d z_{1} \wedge \cdots \wedge d z_{n}
$$

it is normalized so that $d V_{\mathbb{C}}\left(e_{0}, e_{1}, e_{2}, \ldots, e_{n}\right)=1$.

Recall the bilinear form (7) on $\mathbb{C}^{n+1}$.

Definition 7. Let $D_{n} z$ be the unique $\mathbb{C}^{n+1}$-valued complex holomorphic $n$-form on $\mathbb{C}^{n+1}$ such that, for all $Z_{0}, Z_{1}, \ldots, Z_{n} \in \mathbb{C}^{n+1}$, we have

$$
\left\langle Z_{0}, D_{n} z\left(Z_{1}, Z_{2}, \ldots, Z_{n}\right)\right\rangle=d V_{\mathbb{C}}\left(Z_{0}, Z_{1}, Z_{2}, \ldots Z_{n}\right) .
$$

Explicitly, we can express $D_{n} z$ as a sum of $n+1$ terms by substituting basis vectors into (13) yielding

$$
D_{n} z=\sum_{j=0}^{n}(-1)^{j} e_{j} d \hat{z}_{j}
$$

where

$$
d \hat{z}_{j}=d z_{0} \wedge d z_{1} \wedge \cdots \wedge d z_{j-1} \wedge d z_{j+1} \wedge \ldots d z_{n}
$$


Lemma 8. Let $U \subset \mathbb{C}^{n+1}$ be an open set and let $f: U \rightarrow \mathcal{A}_{n}^{\mathbb{C}}, g: U \rightarrow \tilde{M}_{n}^{\mathbb{C}}$ be holomorphic functions, where $\tilde{M}_{n}^{\mathbb{C}}$ is a right $\mathcal{A}_{n}^{\mathbb{C}}$-module. Then,

$$
d\left(g D_{n} z f\right)=\left(g \nabla_{\mathbb{C}}^{+}\right) f d V_{\mathbb{C}}+g\left(\nabla_{\mathbb{C}}^{+} f\right) d V_{\mathbb{C}} \quad \text { and } \quad d\left(g D_{n} z\right)=\left(g \nabla_{\mathbb{C}}^{+}\right) d V_{\mathbb{C}}
$$

Similarly, if $f: U \rightarrow M_{n}^{\mathbb{C}}$ and $g: U \rightarrow \mathcal{A}_{n}^{\mathbb{C}}$ are holomorphic functions, where $M_{n}^{\mathbb{C}}$ is a left $\mathcal{A}_{n}^{\mathbb{C}}$-module,

$$
d\left(g D_{n} z f\right)=\left(g \nabla_{\mathbb{C}}^{+}\right) f d V_{\mathbb{C}}+g\left(\nabla_{\mathbb{C}}^{+} f\right) d V_{\mathbb{C}} \quad \text { and } \quad d\left(D_{n} z f\right)=\left(\nabla_{\mathbb{C}}^{+} f\right) d V_{\mathbb{C}} .
$$

Corollary 9. A holomorphic function $f$ on $U$ is complex left-monogenic if and only if the $n$-form $D_{n} z f$ is closed on $U$. Similarly, a holomorphic function $g$ on $U$ is complex rightmonogenic if and only if the $n$-form $g D_{n} z$ is closed on $U$.

This corollary may be used to give an alternative definition of complex left- and rightmonogenic functions.

Recall that the generators of the real universal Clifford algebra $\mathcal{A}_{p, q}$ and complex universal Clifford algebra $\mathcal{A}_{p+q}^{\mathbb{C}}$ are related by (6) . Therefore, restricting to $\mathbb{R}^{p+q+1}$, we have

$$
\begin{aligned}
& d z_{j}\left(x_{j} e_{j}\right)=x_{j}=d x_{j}\left(x_{j} e_{j}\right), \quad \text { hence }\left.\quad d z_{j}\right|_{\mathbb{R}^{p+q+1}}=d x_{j} \quad \text { if } 0 \leq j \leq p ; \\
& d z_{j}\left(\tilde{x}_{j} \tilde{e}_{j}\right)=i \tilde{x}_{j}=i d \tilde{x}_{j}\left(\tilde{x}_{j} \tilde{e}_{j}\right), \quad \text { hence }\left.\quad d z_{j}\right|_{\mathbb{R}^{p+q+1}}=i d \tilde{x}_{j} \quad \text { if } p+1 \leq j \leq p+q .
\end{aligned}
$$

Definition 10. We define $d V_{p, q}$ to be the restriction of $d V_{\mathbb{C}}$ to $\mathbb{R}^{p+q+1}$ :

$$
d V_{p, q}=\left.d V_{\mathbb{C}}\right|_{\mathbb{R}^{p+q+1}}=i^{q} d x_{0} \wedge \cdots \wedge d x_{p} \wedge d \tilde{x}_{p+1} \wedge \cdots \wedge d \tilde{x}_{p+q}
$$

it is normalized so that $d V_{p, q}\left(e_{0}, e_{1}, \ldots, e_{p}, \tilde{e}_{p+1}, \ldots, \tilde{e}_{p+q}\right)=i^{q}$.

Definition 11. Let $D_{p, q} x$ be the restriction of $D_{p+q} z$ to $\mathbb{R}^{p+q+1}$, so that we have, for all $\left(X_{0}, X_{1}, \ldots, X_{p+q}\right) \in \mathbb{R}^{p+q+1}$,

$$
\left\langle X_{0}, D_{p, q} x\left(X_{1}, X_{2}, \ldots, X_{p+q}\right)\right\rangle=d V_{p, q}\left(X_{0}, X_{1}, \ldots X_{p+q}\right) .
$$

Explicitly,

$$
D_{p, q} x=i^{q}\left(\sum_{j=0}^{p}(-1)^{j} e_{j} d \hat{x}_{j}-\sum_{j=p+1}^{p+q}(-1)^{j} \tilde{e}_{j} d \hat{\tilde{x}}_{j}\right)
$$

where

$$
\begin{array}{ll}
d \hat{x}_{j}=d x_{0} \wedge \cdots \wedge d x_{j-1} \wedge d x_{j+1} \wedge \cdots \wedge d x_{p} \wedge d \tilde{x}_{p+1} \wedge \cdots \wedge d \tilde{x}_{p+q} & \text { if } 0 \leq j \leq p \\
d \tilde{\tilde{x}}_{j}=d x_{0} \wedge \cdots \wedge d x_{p} \wedge d \tilde{x}_{p+1} \wedge \cdots \wedge d \tilde{x}_{j-1} \wedge d \tilde{x}_{j+1} \wedge \cdots \wedge d \tilde{x}_{p+q} & \text { if } p+1 \leq j \leq p+q .
\end{array}
$$

We have the following real analogue of Lemma 8 ; its proof is the same as in the complex case. 
Lemma 12. Let $\underset{\sim}{U} \subset \mathbb{R}^{p+q+1}$ be an open set and let $f: U \rightarrow \mathcal{A}_{p, q}, g: U \rightarrow \tilde{M}_{p, q}$ be $\mathcal{C}^{1}$ functions, where $\tilde{M}_{p, q}$ is a right $\mathcal{A}_{p, q}$-module. Then,

$$
d\left(g D_{p, q} x f\right)=\left(g \nabla_{p, q}^{+}\right) f d V_{p, q}+g\left(\nabla_{p, q}^{+} f\right) d V_{p, q} \quad \text { and } \quad d\left(g D_{p, q} x\right)=\left(g \nabla_{p, q}^{+}\right) d V_{p, q} .
$$

Similarly, if $f: U \rightarrow M_{p, q}$ and $g: U \rightarrow \mathcal{A}_{p, q}$ are $\mathcal{C}^{1}$ functions, where $M_{p, q}$ is a left $\mathcal{A}_{p, q^{-}}$ module,

$$
d\left(g D_{p, q} x f\right)=\left(g \nabla_{p, q}^{+}\right) f d V_{p, q}+g\left(\nabla_{p, q}^{+} f\right) d V_{p, q} \quad \text { and } \quad d\left(D_{p, q} x f\right)=\left(\nabla_{p, q}^{+} f\right) d V_{p, q} .
$$

Corollary 13. $A \mathcal{C}^{1}$ function $f$ on $U$ is $(p, q)$-left-monogenic if and only if the $n$-form $D_{p, q} x f$ is closed on $U$. Similarly, a $\mathcal{C}^{1}$ function $g$ on $U$ is $(p, q)$-right-monogenic if and only if the $n$-form $g D_{p, q} x$ is closed on $U$.

Again, this corollary may serve as an alternative definition of $(p, q)$-left- and $(p, q)$-rightmonogenic functions.

We orient $\mathbb{R}^{p+q+1} \subset \mathcal{A}_{p, q}$ so that

$$
\left\{e_{0}, e_{1}, e_{2}, \ldots, e_{p}, \tilde{e}_{p+1}, \ldots, \tilde{e}_{p+q}\right\}
$$

is a positively oriented basis. For an open subset $U \subset \mathbb{R}^{p+q+1}$ with piecewise smooth boundary $\partial U$, at each smooth boundary point $X \in \partial U$, let

$$
\mathbf{n}_{X}=n_{0} e_{0}+n_{1} e_{1}+\cdots+n_{p} e_{p}+\tilde{n}_{p+1} \tilde{e}_{p+1}+\cdots+\tilde{n}_{p+q} \tilde{e}_{p+q}
$$

be an outward pointing normal unit vector to $U$ at $X$ (with respect to (9)). Then we can orient the boundary $\partial U$ so that a basis $\left\{v_{1}, \ldots, v_{p+q}\right\}$ of the tangent space $T_{X} U$ is positively oriented if and only if $\left\{\mathbf{n}_{X}, v_{1}, \ldots, v_{p+q}\right\}$ is a positively oriented basis of $\mathbb{R}^{p+q+1}$. Let $d S_{p, q}$ be the contraction of $\mathbf{n}_{X}$ with $d V_{p, q}$, so that

$$
d S_{p, q}\left(v_{1}, \ldots, v_{p+q}\right)=d V_{p, q}\left(\mathbf{n}_{X}, v_{1}, \ldots, v_{p+q}\right)
$$

whenever $v_{1}, \ldots, v_{p+q}$ are vectors in the tangent space $T_{X} U$ of $\partial U$ at $X$. Explicitly,

$$
d S_{p, q}=i^{q}\left(\sum_{j=0}^{p}(-1)^{j} n_{j} d \hat{x}_{j}+\sum_{j=p+1}^{p+q}(-1)^{j} n_{j} d \hat{\tilde{x}}_{j}\right) .
$$

Lemma 14. With $\overline{\mathbf{n}}_{X}$ denoting the complex conjugate of $\mathbf{n}_{X}$ (recall (8)), the restriction

$$
\left.D_{p, q} x\right|_{\partial U}=\overline{\mathbf{n}}_{X} d S_{p, q}
$$

Proof. By definition, $\mathbf{n}_{X}$ is orthogonal to the tangent space $T_{X} \partial U$. Thus, for every vector $X=x_{0} e_{0}+x_{1} e_{1}+\cdots+x_{p} e_{p}+\tilde{x}_{p+1} \tilde{e}_{p+1}+\cdots+\tilde{x}_{p+q} \tilde{e}_{p+q}$ in $T_{X} \partial U$, we have

$$
\sum_{j=0}^{p} n_{j} x_{j}+\sum_{j=p+1}^{p+q} \tilde{n}_{j} \tilde{x}_{j}=0
$$


and hence

$$
n_{0} d x_{0}+\cdots+n_{p} d x_{p}+\tilde{n}_{p+1} d \tilde{x}_{p+1}+\cdots+\tilde{n}_{p+q} d \tilde{x}_{p+q}=0 .
$$

Without loss of generality we may suppose $n_{0} \neq 0$, then we can isolate $d x_{0}$ :

$$
d x_{0}=-\frac{1}{n_{0}}\left(n_{1} d x_{1}+n_{2} d x_{2}+\cdots+n_{p} d x_{p}+\tilde{n}_{p+1} d \tilde{x}_{p+1}+\cdots+\tilde{n}_{p+q} d \tilde{x}_{p+q}\right) .
$$

We introduce the symbol $d \check{x}_{j}$ denoting wedge products of all but $d x_{0}, d x_{j}$ :

$$
\begin{array}{ll}
d \check{x}_{j}=d x_{1} \wedge \cdots \wedge d x_{j-1} \wedge d x_{j+1} \wedge \cdots \wedge d x_{p} \wedge d \tilde{x}_{p+1} \wedge \cdots \wedge d \tilde{x}_{p+q} & \text { if } 1 \leq j \leq p \\
d \tilde{\tilde{x}}_{j}=d x_{1} \wedge \cdots \wedge d x_{p} \wedge d \tilde{x}_{p+1} \wedge \cdots \wedge d \tilde{x}_{j-1} \wedge d \tilde{x}_{j+1} \wedge \cdots \wedge d \tilde{x}_{p+q} & \text { if } p+1 \leq j \leq p+q .
\end{array}
$$

Then

$$
\begin{array}{ll}
d x_{j} \wedge d \check{x}_{j}=(-1)^{j-1} d \hat{x}_{0} & \text { if } 1 \leq j \leq p, \\
d \tilde{x}_{j} \wedge d \check{\tilde{x}}_{j}=(-1)^{j-1} d \hat{x}_{0} & \text { if } p+1 \leq j \leq p+q .
\end{array}
$$

Substituting (19) into the expression (18) for $d S_{p, q}$ and using these notations,

$$
\begin{aligned}
d S_{p, q}=i^{q}\left(n_{0} d \hat{x}_{0}+\sum_{j=1}^{p}(-1)^{j}\left(-\frac{n_{j}^{2}}{n_{0}}\right) d x_{j} \wedge d \check{x}_{j}\right)+ & \left.\sum_{j=p+1}^{p+q}(-1)^{j}\left(-\frac{\tilde{n}_{j}^{2}}{n_{0}}\right) d \tilde{x}_{j} \wedge d \check{x}_{j}\right) \\
& =\frac{i^{q}}{n_{0}}\left(\sum_{j=0}^{p} n_{j}^{2}+\sum_{j=p+1}^{p+q} \tilde{n}_{j}^{2}\right) d \hat{x}_{0}=\frac{i^{q}}{n_{0}} d \hat{x}_{0}
\end{aligned}
$$

since $\mathbf{n}_{X}$ is a unit vector. We perform a similar computation for $D_{p, q} x$ substituting (19) into (17) to get

$$
\begin{aligned}
&\left.D_{p, q} x\right|_{\partial U}=i^{q}\left(\sum_{j=0}^{p}(-1)^{j} e_{j} d \hat{x}_{j}\right.\left.-\sum_{j=p+1}^{p+q}(-1)^{j} \tilde{e}_{j} d \hat{\tilde{x}}_{j}\right) \\
&=i^{q}\left(n_{0} d \hat{x}_{0}+\sum_{j=1}^{p}(-1)^{j}\left(-\frac{n_{j}}{n_{0}}\right) e_{j} d x_{j} \wedge d \check{x}_{j}-\sum_{j=p+1}^{p+q}(-1)^{j}\left(-\frac{\tilde{n}_{j}}{n_{0}}\right) \tilde{e}_{j} d \tilde{x}_{j} \wedge d \check{\tilde{x}}_{j}\right) \\
&=\frac{i^{q}}{n_{0}}\left(\sum_{j=0}^{p} n_{j} e_{j}-\sum_{j=p+1}^{p+q} \tilde{n}_{j} \tilde{e}_{j}\right) d \hat{x}_{0}=\frac{i^{q}}{n_{0}} \overline{\mathbf{n}}_{X} d \hat{x}_{0}=\overline{\mathbf{n}}_{X} d S_{p, q} .
\end{aligned}
$$

Let $K_{r}$ and $S_{r}$ be the boundaries of the sets $\left\{X \in \mathbb{R}^{p+q+1} ; N(X) \leq r\right\}$ and $\{X \in$ $\left.\mathbb{R}^{p+q+1} ;\|X\|^{2} \leq r\right\}$ respectively. Note that the outward pointing normal vectors at $X$ will be respectively $\bar{X} /\|X\|$ and $X /\|X\|$, so we get the following analogue of Lemma 3 of $[\mathrm{L}]$ :

\section{Corollary 15.}

$$
\left.D_{p, q} x\right|_{K_{r}}=\frac{X}{\|X\|} d S_{p, q},\left.\quad D_{p, q} x\right|_{S_{r}}=\frac{\bar{X}}{\|X\|} d S_{p, q}=\frac{\bar{X}}{r} d S_{p, q} .
$$


These two expressions are the same in the positive definite case (when $q=0$ ).

We state the classical Cauchy's integral formulas for left- and right-monogenic functions in the positive definite case $(q=0)$. For details and proofs see, for example, [BDS, DSS, GM]. Recall that in this case we write $\mathcal{A}_{n}$ instead of $\mathcal{A}_{n, 0}$.

Theorem 16. Let $n \geq 2$. Let $U \subset \mathbb{R}^{n+1} \subset \mathcal{A}_{n}$ be an open bounded set with piecewise $\mathcal{C}^{1}$ boundary $\partial U$, and let $M_{n}$ be a left $\mathcal{A}_{n}$-module. Suppose that an $M_{n}$-valued function $f$ is $(n, 0)$-left-monogenic function on a neighborhood of the closure $\bar{U}$. We have:

$$
\int_{\partial U} G_{n, 0}\left(X-X_{0}\right) D_{n, 0} x f(X)= \begin{cases}\omega_{n} f\left(X_{0}\right) & \text { if } X_{0} \in U, \\ 0 & \text { if } X_{0} \notin \bar{U}\end{cases}
$$

where $\omega_{n}$ denotes the $n$-dimensional volume of the unit $n$-sphere in $\mathbb{R}^{n+1}$.

Similarly, if $\tilde{M}_{n}$ is a right $\mathcal{A}_{n}$-module and $g$ is an $\tilde{M}_{n}$-valued function that is $(n, 0)$-rightmonogenic on a neighborhood of $\bar{U}$, then

$$
\int_{\partial U} g(X) D_{n, 0} x G_{n, 0}\left(X-X_{0}\right)= \begin{cases}\omega_{n} g\left(X_{0}\right) & \text { if } X_{0} \in U, \\ 0 & \text { if } X_{0} \notin \bar{U} .\end{cases}
$$

\section{$5 \quad$ First Cauchy's Integral Formula}

In this section we present an integral formula for $(p, q)$-monogenic functions that have complex holomorphic extension to some open neighborhood in $\mathbb{C}^{p+q+1}$. The statement of the formula involves a one-parameter deformation map $h_{\varepsilon}$ that is used to deform the contour of integration so that it does not cross the singularities of $N\left(X-X_{0}\right)^{-\frac{p+q+1}{2}}$.

Definition 17. For $0 \leq \varepsilon \leq 1$, let $h_{\varepsilon}: \mathbb{C}^{p+q+1} \rightarrow \mathbb{C}^{p+q+1}$ be a linear map defined by

$$
Z=\sum_{j=0}^{p+q} z_{j} e_{j} \quad \mapsto \quad h_{\varepsilon}(Z)=\sum_{j=0}^{p}(1+i \varepsilon) z_{j} e_{j}+\sum_{j=p+1}^{q+1}(1-i \varepsilon) z_{j} e_{j}
$$

Note that the map $h_{\varepsilon}$ depends on $p$ and $q$, but we suppress this dependence in order to avoid cumbersome notations.

Corollary 18. If $X=\sum_{j=0}^{p} x_{j} e_{j}+\sum_{j=p+1}^{p+q} \tilde{x}_{j} \tilde{e}_{j} \in \mathbb{R}^{p+q+1} \subset \mathcal{A}_{p, q} \subset \mathcal{A}_{p+q}^{\mathbb{C}}$, then

$$
N\left(h_{\varepsilon}(X)\right)=\left(1-\varepsilon^{2}\right) N(X)+2 i \varepsilon\|X\|^{2} .
$$

In particular, when $\varepsilon=1$,

$$
N\left(h_{1}(X)\right)=2 i\|X\|^{2} .
$$

Definition 19. For $Z_{0} \in \mathbb{C}^{p+q+1}$ fixed, we define

$$
h_{\varepsilon, Z_{0}}: Z \quad \mapsto \quad Z_{0}+h_{\varepsilon}\left(Z-Z_{0}\right)
$$


Theorem 20. Let $p+q \geq 2$. Let $U \subset \mathbb{R}^{p+q+1} \subset \mathcal{A}_{p, q}$ be an open bounded set with piecewise $\mathcal{C}^{1}$ boundary $\partial U$, and let $M_{p+q}^{\mathbb{C}}$ be a left $\mathcal{A}_{p+q}^{\mathbb{C}}$-module. Suppose that an $M_{p+q}^{\mathbb{C}}$-valued function $f$ is $(p, q)$-left-monogenic on a neighborhood of the closure $\bar{U}$. Furthermore, suppose $f$ extends to a complex left-monogenic function $f^{\mathbb{C}}: W^{\mathbb{C}} \rightarrow M_{p+q}^{\mathbb{C}}$, with $W^{\mathbb{C}} \subset \mathbb{C}^{p+q+1} \subset \mathcal{A}_{p+q}^{\mathbb{C}}$ an open subset containing $\bar{U}$. We have:

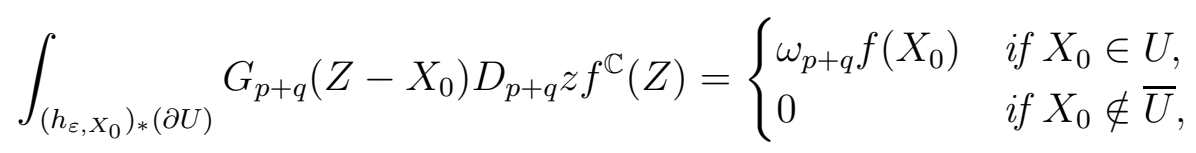

for all $\varepsilon>0$ sufficiently close to 0 .

Similarly, if $\tilde{M}_{p+q}^{\mathbb{C}}$ is a right $\mathcal{A}_{p+q}^{\mathbb{C}}$-module and $g$ is an $\tilde{M}_{p+q}^{\mathbb{C}}$-valued function that is $(p, q)$ right-monogenic on a neighborhood of $\bar{U}$ and can be extended to a complex right-monogenic function $g^{\mathbb{C}}: W^{\mathbb{C}} \rightarrow \tilde{M}_{p+q}^{\mathbb{C}}$, then

$$
\int_{\left(h_{\varepsilon}, X_{0}\right)_{*}(\partial U)} g^{\mathbb{C}}(Z) D_{p+q} z G_{p+q}\left(Z-X_{0}\right)= \begin{cases}\omega_{p+q} g\left(X_{0}\right) & \text { if } X_{0} \in U, \\ 0 & \text { if } X_{0} \notin \bar{U},\end{cases}
$$

for all $\varepsilon>0$ sufficiently close to 0 .

Remark 21. For all $\varepsilon \neq 0$ sufficiently close to 0 the contour of integration $\left(h_{\varepsilon, X_{0}}\right)_{*}(\partial U)$ lies inside $W^{\mathbb{C}}$ and the integrand is non-singular, thus the integrals are well-defined. Moreover, we will see that the value of the integral becomes constant when the parameter $\varepsilon$ is sufficiently close to 0 .

Remark 22. If we require $\varepsilon<0$, then we need to insert a factor of $(-1)^{q}$ into the right hand sides of the above formulas. Thus,

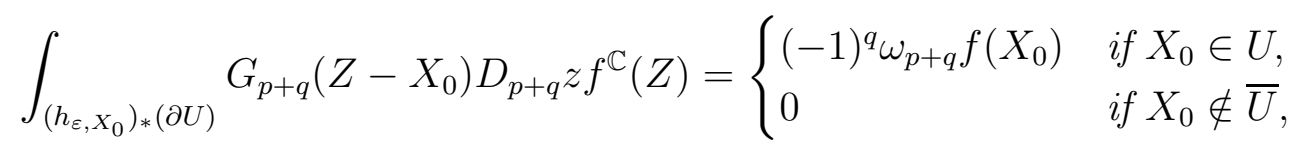

for all $\varepsilon<0$ sufficiently close to 0 . And similarly for the other formula.

Proof. We consider only the left-monogenic case, the right-monogenic case is similar. By translation, we may assume that $X_{0}=0$. Let $M=\sup _{X \in \partial U}\|X\|$. We restrict $W^{\mathbb{C}}$ to be the $\delta$-neighborhood of $\bar{U}$ in $\mathbb{C}^{p+q+1}$ for some sufficiently small $\delta>0$. Consider $0<|\varepsilon|<\delta / M$. If $X \in \mathbb{R}^{p+q+1} \backslash\{0\}$, then, by (20), $N\left(h_{\varepsilon}(X)\right)$ is not a negative real, hence $h_{\varepsilon}(X) \in \mathbb{C}_{G}^{p+q+1}$. Thus, we have that $\left(h_{\varepsilon}\right)_{*}(\partial U)$ lies inside $\mathbb{C}_{G}^{p+q+1} \cap W^{\mathbb{C}}$. By Lemma 8 , the integrand is a closed form in the region $\mathbb{C}_{G}^{p+q+1} \cap W^{\mathbb{C}}$, so the integral stays unchanged for all $-\delta / M<\varepsilon<0$ and $0<\varepsilon<\delta / M$ (but the values of the integral may be different on these two intervals). If $X_{0}=0 \notin \bar{U}$, we have that $\left(h_{\varepsilon}\right)_{*}(\bar{U}) \subseteq \mathbb{C}_{G}^{p+q+1} \cap W^{\mathbb{C}}$, the integrand is a closed form, and the integral over $\partial U$ is zero, thus we are done.

Now, suppose $X_{0}=0 \in U$. Choose an $r>0$ sufficiently small such that $r<\delta / 2$ and the ball $B_{r}=\left\{X \in \mathbb{R}^{p+q+1}:\|X\| \leq r\right\}$ is contained in $U$. We orient the sphere $S_{r}=\left\{X \in \mathbb{R}^{p+q+1}:\|X\|=r\right\}$ as the boundary of $B_{r}$. By Stokes' Theorem and (15) we 
have:

$$
\begin{aligned}
& \int_{\left(h_{\varepsilon}\right)_{*}(\partial U)} G_{p+q}(Z) D_{p+q} z f^{\mathbb{C}}(Z)-\int_{\left(h_{\varepsilon}\right)_{*} S_{r}} G_{p+q}(Z) D_{p+q} z f^{\mathbb{C}}(Z) \\
&=\int_{\left(h_{\varepsilon}\right)_{*}\left(U \backslash B_{r}\right)} d\left(G_{p+q}(Z) D_{p+q} z f^{\mathbb{C}}(Z)\right)=\int_{\left(h_{\varepsilon}\right)_{*}\left(U \backslash B_{r}\right)} 0 d V_{\mathbb{C}}=0 .
\end{aligned}
$$

Hence,

$$
\int_{\left(h_{\varepsilon}\right)_{*}(\partial U)} G_{p+q}(Z) D_{p+q} z f^{\mathbb{C}}(Z)=\int_{\left(h_{\varepsilon}\right)_{*} S_{r}} G_{p+q}(Z) D_{p+q} z f^{\mathbb{C}}(Z) .
$$

By varying the parameter $\varepsilon$ from its original value to 1 , we can continuously deform $\left(h_{\varepsilon}\right)_{*} S_{r}$ into $\left(h_{1}\right)_{*}\left(S_{r}\right)$ within $\mathbb{C}_{G}^{p+q+1} \cap W^{\mathbb{C}}$ without changing the value of the integral (here we are using $r<\delta / 2)$, and so we have:

$$
\int_{\left(h_{\varepsilon}\right)_{*}(\partial U)} G_{p+q}(Z) D_{p+q} z f^{\mathbb{C}}(Z)=\int_{\left(h_{1}\right)_{*} S_{r}} G_{p+q}(Z) D_{p+q} z f^{\mathbb{C}}(Z) .
$$

Next, we rotate the sphere $\left(h_{1}\right)_{*} S_{r}$ using the map $Z \mapsto e^{-i t} Z, 0 \leq t \leq \pi / 4$. Since $N\left(e^{-i t} Z\right)=e^{-2 i t} N(Z)$ and $\left\|e^{-i t} Z\right\|=\|Z\|$, as $t$ varies continuously from 0 to $\pi / 4$, the sphere $\left(h_{1}\right)_{*} S_{r}$ gets rotated inside $\mathbb{C}_{G}^{p+q+1} \cap W^{\mathbb{C}}$ into $\tilde{S}_{r \sqrt{2}}$ - the sphere of radius $r \sqrt{2}$ contained in $\operatorname{Span}\left\{e_{0}, e_{1}, \ldots, e_{p+q}\right\}=\mathbb{R}^{p+q+1} \subset \mathcal{A}_{p+q, 0}$. Thus, we have by Stokes' Theorem and the classical integral formula for the definite case (Theorem 16):

$$
\begin{aligned}
\int_{\left(h_{\varepsilon}\right)_{*}(\partial U)} G_{p+q}(Z) D_{p+q} z f^{\mathbb{C}}(Z)=\int_{\tilde{S}_{r \sqrt{2}}} G_{p+q}(Z) D_{p+q} z f^{\mathbb{C}}(Z) \\
\quad=\int_{\tilde{S}_{r \sqrt{2}}} G_{p+q, 0}(X) D_{p+q, 0} x f^{\mathbb{C}}(X)=\omega_{p+q} f(0) .
\end{aligned}
$$

\section{Second Cauchy's Integral Formula}

In this section we state and prove another integral formula for $(p, q)$-monogenic functions. Unlike the previous version, this one that does not change the contour of integration. Instead, we insert a purely imaginary term $i \varepsilon\left\|X-X_{0}\right\|^{2}$ into the denominator of the reproducing kernel $G_{p, q}\left(X-X_{0}\right)$, then take limit as $\varepsilon \rightarrow 0^{+}$.

Theorem 23. Let $p+q \geq 2$. Let $U \subset \mathbb{R}^{p+q+1}$ be a bounded open region with smooth boundary $\partial U$, and let $M_{p, q}$ be a left $\mathcal{A}_{p, q}$-module. Suppose that $f$ is an $M_{p, q}$-valued function that is $(p, q)$-left-monogenic in a neighborhood of the closure $\bar{U}$. Then, for any $X_{0} \in \mathbb{R}^{p+q+1}$ such that $\partial U$ intersects the cone $\left\{X \in \mathbb{R}^{p+q+1} ; N\left(X-X_{0}\right)=0\right\}$ transversally, we have:

$$
\lim _{\varepsilon \rightarrow 0^{+}} \int_{\partial U} G_{p, q, \varepsilon}\left(X-X_{0}\right) D_{p, q} x f(X)= \begin{cases}\omega_{p+q} f\left(X_{0}\right) & \text { if } X_{0} \in U, \\ 0 & \text { if } X_{0} \notin \bar{U}\end{cases}
$$


where $G_{p, q, \varepsilon}$ is the modified Green's function defined by

$$
\begin{aligned}
G_{p, q, \varepsilon}(X)= & \frac{X^{+}}{\left(N(X)+i \varepsilon\|X\|^{2}\right)^{\frac{p+q+1}{2}}} \\
& =\frac{x_{0}-\sum_{j=1}^{p} x_{j} e_{j}-\sum_{j=p+1}^{p+q} \tilde{x}_{j} \tilde{e}_{j}}{\left(\sum_{j=0}^{p} x_{j}^{2}-\sum_{j=p+1}^{p+q} \tilde{x}_{j}^{2}+i \varepsilon\left(\sum_{j=0}^{p} x_{j}^{2}+\sum_{j=p+1}^{p+q} \tilde{x}_{j}^{2}\right)\right)^{\frac{p+q+1}{2}}} .
\end{aligned}
$$

Similarly, if $\tilde{M}_{p, q}$ is a right $\mathcal{A}_{p, q}$-module and $g$ is an $\tilde{M}_{p, q}$-valued function that is $(p, q)$ right-monogenic in a neighborhood of $\bar{U}$, then, for any $X_{0} \in \mathbb{R}^{p+q+1}$ such that $\partial U$ intersects the cone $\left\{X \in \mathbb{R}^{p+q+1} ; N\left(X-X_{0}\right)=0\right\}$ transversally, we have:

$$
\lim _{\varepsilon \rightarrow 0^{+}} \int_{\partial U} g(X) D_{p, q} x G_{p, q, \varepsilon}\left(X-X_{0}\right)= \begin{cases}\omega_{p+q} g\left(X_{0}\right) & \text { if } X_{0} \in U, \\ 0 & \text { if } X_{0} \notin \bar{U} .\end{cases}
$$

Remark 24. As in the case of the first Cauchy's integral formula (Theorem 20), if we let $\varepsilon \rightarrow 0^{-}$, we need to insert a factor of $(-1)^{q}$ into the right hand sides of the above formulas. Thus,

$$
\lim _{\varepsilon \rightarrow 0^{-}} \int_{\partial U} G_{p, q, \varepsilon}\left(X-X_{0}\right) D_{p, q} x f(X)= \begin{cases}(-1)^{q} \omega_{p+q} f\left(X_{0}\right) & \text { if } X_{0} \in U, \\ 0 & \text { if } X_{0} \notin \bar{U}\end{cases}
$$

And similarly for the other formula.

Proof. We change coordinates to hybrid spherical coordinates given by

$$
\begin{aligned}
& \Phi\left(\rho, \varphi_{1}, \ldots, \varphi_{p}, \theta, \psi_{1}, \ldots, \psi_{q-1}\right)=\left(x_{0}, x_{1}, \ldots, x_{p}, \tilde{x}_{p+1}, \ldots, \tilde{x}_{p+q}\right), \\
& x_{0}=\rho \cos \theta \cos \varphi_{1} \\
& x_{1}=\rho \cos \theta \sin \varphi_{1} \cos \varphi_{2} \\
& x_{2}=\rho \cos \theta \sin \varphi_{1} \sin \varphi_{2} \cos \varphi_{3} \\
& \vdots \\
& x_{p-1}=\rho \cos \theta \sin \varphi_{1} \ldots \cos \varphi_{p} \quad 0 \leq \varphi_{1}, \varphi_{2}, \ldots, \varphi_{p-1} \leq \pi, \\
& x_{p}=\rho \cos \theta \sin \varphi_{1} \ldots \sin \varphi_{p} \quad 0 \leq \psi_{1}, \psi_{2}, \ldots, \psi_{q-2} \leq \pi, \\
& \tilde{x}_{p+1}=\rho \sin \theta \cos \psi_{1} \quad 0 \leq \varphi_{p}, \psi_{q-1} \leq 2 \pi, \\
& \tilde{x}_{p+2}=\rho \sin \theta \sin \psi_{1} \cos \psi_{2} \quad 0 \leq \rho, \quad 0 \leq \theta \leq \frac{\pi}{2} . \\
& \vdots \\
& \tilde{x}_{p+q-1}=\rho \sin \theta \sin \psi_{1} \ldots \cos \psi_{q-1} \\
& \tilde{x}_{p+q}=\rho \sin \theta \sin \psi_{1} \ldots \sin \psi_{q-1}
\end{aligned}
$$

These are essentially the spherical coordinates for the $\left(x_{0}, \ldots, x_{p}\right)$-subspace combined with the spherical coordinates for the $\left(\tilde{x}_{p+1}, \ldots, \tilde{x}_{p+q}\right)$-subspace. In these coordinates, we have:

$$
\begin{aligned}
& N(X)=\sum_{j=0}^{p} x_{j}^{2}-\sum_{j=p+1}^{p+q} \tilde{x}_{j}^{2}=\rho^{2} \cos ^{2} \theta-\rho^{2} \sin ^{2} \theta=\rho^{2} \cos (2 \theta) \quad \text { and } \\
& \|X\|^{2}=\sum_{j=0}^{p} x_{j}^{2}+\sum_{j=p+1}^{p+q} \tilde{x}_{j}^{2}=\rho^{2} \cos ^{2} \theta+\rho^{2} \sin ^{2} \theta=\rho^{2} .
\end{aligned}
$$


We thus have that the null cone $\mathcal{N}_{p, q}$ is the set of $X$ in $\mathbb{R}^{p+q+1}$ such that $\theta=\frac{\pi}{4}$, and we structure our argument in the vein of $[\mathrm{L}$. We use the symmetry of the change of basis matrix with respect to $p$ and $q-1$ to calculate the determinant by means of block matrices. Let $S_{n, \alpha}$ be the Jacobian matrix corresponding to the transformation into the standard $(n+1)$ dimensional spherical coordinates, $\left(\rho, \alpha_{1}, \ldots, \alpha_{n}\right) \rightarrow\left(x_{0}, \ldots, x_{n}\right) \in \mathbb{R}^{n+1}$,

$$
\operatorname{det}\left(S_{n, \alpha}\right)=\rho^{n} \sin ^{n-1} \alpha_{1} \sin ^{n-2} \alpha_{2} \ldots \sin \alpha_{n-1} \text {. }
$$

Lemma 25. The determinant of the Jacobian matrix of the change of coordinates (21) is given by

$$
\operatorname{det}(D \Phi)=\rho \cos ^{p} \theta \sin ^{q-1} \theta \operatorname{det}\left(S_{p, \varphi}\right) \operatorname{det}\left(S_{q-1, \psi}\right) .
$$

Proof. We spell out the Jacobian matrix of the change of coordinates (21) and divide it into blocks as follows:

$$
D \Phi=\left(\begin{array}{ccccc|cccc}
\frac{\partial x_{0}}{\partial \rho} & \frac{\partial x_{0}}{\partial \varphi_{1}} & \frac{\partial x_{0}}{\partial \varphi_{2}} & \ldots & \frac{\partial x_{0}}{\partial \varphi_{p}} & \frac{\partial x_{0}}{\partial \theta} & \frac{\partial x_{0}}{\partial \psi_{1}} & \ldots & \frac{\partial x_{0}}{\partial \psi_{q-1}} \\
\frac{\partial x_{1}}{\partial \rho} & \frac{\partial x_{1}}{\partial \varphi_{1}} & \frac{\partial x_{1}}{\partial \varphi_{2}} & \ldots & \frac{\partial x_{1}}{\partial \varphi_{p}} & \frac{\partial x_{1}}{\partial \theta} & \frac{\partial x_{1}}{\partial \psi_{1}} & \ldots & \frac{\partial x_{1}}{\partial \psi_{q-1}} \\
\vdots & \vdots & \vdots & \ddots & \vdots & \vdots & \vdots & \ddots & \vdots \\
\frac{\partial x_{p}}{\partial \rho} & \frac{\partial x_{p}}{\partial \varphi_{1}} & \frac{\partial x_{p}}{\partial \varphi_{2}} & \ldots & \frac{\partial x_{p}}{\partial \varphi_{p}} & \frac{\partial x_{p}}{\partial \theta} & \frac{\partial x_{p}}{\partial \psi_{1}} & \ldots & \frac{\partial x_{p}}{\partial \psi_{q-1}} \\
\hline \frac{\partial \tilde{x}_{p+1}}{\partial \rho} & \frac{\partial \tilde{x}_{p+1}}{\partial \varphi_{1}} & \frac{\partial \tilde{x}_{p+1}}{\partial \varphi_{2}} & \ldots & \frac{\partial \tilde{x}_{p+1}}{\partial \varphi_{p}} & \frac{\partial \tilde{x}_{p+1}}{\partial \theta} & \frac{\partial \tilde{x}_{p+1}}{\partial \psi_{1}} & \ldots & \frac{\partial \tilde{x}_{p+1}}{\partial \psi_{q-1}} \\
\vdots & \vdots & \vdots & \ddots & \vdots & \vdots & \vdots & \ddots & \vdots \\
\frac{\partial \tilde{x}_{p+q}}{\partial \rho} & \frac{\partial \tilde{x}_{p+q}}{\partial \varphi_{1}} & \frac{\partial \tilde{x}_{p+q}}{\partial \varphi_{2}} & \ldots & \frac{\partial \tilde{x}_{p+q}}{\partial \varphi_{p}} & \frac{\partial \tilde{x}_{p+q}}{\partial \theta} & \frac{\partial \tilde{x}_{p+q}}{\partial \psi_{1}} & \ldots & \frac{\partial \tilde{x}_{p+q}}{\partial \psi_{q-1}}
\end{array}\right)=\left(\begin{array}{cc}
A & B \\
\Gamma & \Delta
\end{array}\right) .
$$

We make the following observations about the blocks $A, B, \Gamma$ and $\Delta$. First, $A$ is the $(p+1) \times(p+1)$ matrix that is obtained from $S_{p, \varphi}$ by multiplying each entry by $\cos \theta$; we denote the first column of $A$ by $\vec{a}$. Next, $B$ is the $(p+1) \times q$ matrix whose first column is $\vec{b}$, described below, and all other columns are zero. Similarly, $\Gamma$ is the $q \times(p+1)$ matrix whose first column is $\vec{c}$, described below, and the rest of the columns are zero. Finally, $\Delta$ the $q \times q$ matrix obtained from $S_{q-1, \psi}$ by multiplying the first column by $\rho \cos \theta$ and multiplying the remaining columns by $\sin \theta$; we denote the first column of $\Delta$ by $\vec{d}$.

$$
\begin{gathered}
A=\cos \theta \cdot S_{p, \varphi}, \quad \Delta=S_{q-1, \psi} \cdot\left(\begin{array}{cccc}
\rho \cos \theta & 0 & 0 & \ldots \\
0 & \sin \theta & 0 & \ldots \\
0 & 0 & \sin \theta & \ldots \\
0 & 0 & 0 & \ddots
\end{array}\right), \\
\vec{a}=\cos \theta \cdot\left(\begin{array}{c}
\cos \varphi_{1} \\
\sin \varphi_{1} \cos \varphi_{2} \\
\vdots \\
\sin \varphi_{1} \sin \varphi_{2} \ldots \cos \varphi_{p} \\
\sin \varphi_{1} \sin \varphi_{2} \ldots \sin \varphi_{p}
\end{array}\right), \quad \vec{b}=-\frac{\rho \sin \theta}{\cos \theta} \cdot \vec{a},
\end{gathered}
$$




$$
\vec{c}=\sin \theta \cdot\left(\begin{array}{c}
\cos \psi_{1} \\
\sin \psi_{1} \cos \psi_{2} \\
\vdots \\
\sin \psi_{1} \sin \psi_{2} \ldots \cos \psi_{q-1} \\
\sin \psi_{1} \sin \psi_{2} \ldots \sin \psi_{q-1}
\end{array}\right), \quad \vec{d}=\frac{\rho \cos \theta}{\sin \theta} \cdot \vec{c}
$$

Lemma 26. If $A$ is an $m \times m$ matrix, $B$ is an $m \times n$ matrix, $\Gamma$ is an $n \times m$ matrix, and $\Delta$ is an invertible $n \times n$ matrix, then $\operatorname{det}\left(\begin{array}{cc}A & B \\ \Gamma & \Delta\end{array}\right)=\operatorname{det}\left(A-B \Delta^{-1} \Gamma\right) \operatorname{det}(\Delta)$.

Proof. This standard result on block matrices is proved by factoring into triangular matrices:

$$
\left(\begin{array}{cc}
A & B \\
\Gamma & \Delta
\end{array}\right)=\left(\begin{array}{cc}
A-B \Delta^{-1} \Gamma & B \Delta^{-1} \\
0_{n, m} & I_{n, n}
\end{array}\right)\left(\begin{array}{cc}
I_{m, m} & 0_{m, n} \\
\Gamma & \Delta
\end{array}\right)
$$

Using this lemma,

$$
\operatorname{det}(D \Phi)=\operatorname{det}\left(\begin{array}{ll}
A & B \\
\Gamma & \Delta
\end{array}\right)=\operatorname{det}\left(A-B \Delta^{-1} \Gamma\right) \operatorname{det}(\Delta)
$$

We next calculate $A-B \Delta^{-1} \Gamma$. We first compute $B^{-1} \Delta \Gamma$, labelling the $j$ th row of the matrix $\Delta^{-1}$ by $\overrightarrow{r_{j}}$ (horizontal vector),

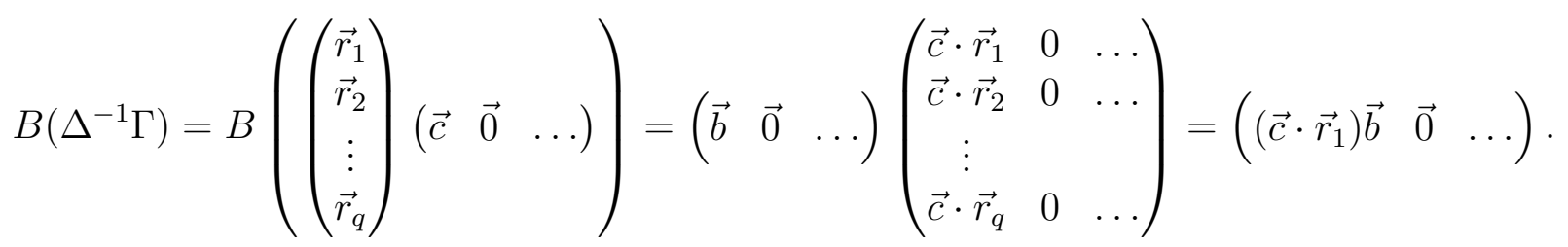

Observe that $\vec{c}$ is the first column of $\Delta$ scaled by $\frac{\sin \theta}{\rho \cos \theta}$, and the so the scalar product of it and the first row of $\Delta^{-1}$ is $\frac{\sin \theta}{\rho \cos \theta}$, as 1 is the upper left entry of $\Delta \Delta^{-1}$. Thus, we have that $B \Delta^{-1} \Gamma$ is a matrix with first column $\frac{\sin \theta}{\rho \cos \theta} \vec{b}$, and the rest of columns zero. Since $\vec{b}=-\frac{\rho \sin \theta}{\cos \theta} \vec{a}$, we have that $A-B \Delta^{-1} \Gamma$ is matrix $A$ with the first column multiplied by $1+\frac{\sin ^{2} \theta}{\cos ^{2} \theta}=\frac{1}{\cos ^{2} \theta}$. Using the multilinearity of the determinant, and factoring out the $\cos \theta$ and $\sin \theta$ from the columns of $A$ and $\Delta$,

$$
\begin{aligned}
\operatorname{det}(D \Phi)=\operatorname{det}( & \left.A-B \Delta^{-1} \Gamma\right) \operatorname{det}(\Delta)=\frac{1}{\cos ^{2} \theta} \operatorname{det}(A) \operatorname{det}(\Delta) \\
=\frac{1}{\cos ^{2} \theta}\left(\cos ^{p+1} \theta \cdot \rho \cos \theta \cdot \sin ^{q-1} \theta\right) \operatorname{det}\left(S_{p, \varphi}\right) \operatorname{det}\left(S_{q-1, \psi}\right) & =\rho \cos ^{p} \theta \sin ^{q-1} \theta \operatorname{det}\left(S_{p, \varphi}\right) \operatorname{det}\left(S_{q-1, \psi}\right) .
\end{aligned}
$$

We return to the proof of Theorem 23. The case $X_{0} \notin \bar{U}$ is easier, so we assume $X_{0} \in U$. By translation we can also assume that $X_{0}=0$. This lemma is proved by direct computation. 
Lemma 27. We have:

$$
\begin{aligned}
& \nabla_{p, q}^{+} G_{p, q, \varepsilon}=i \varepsilon(p+q+1) \frac{\|X\|^{2}-\bar{X} X^{+}}{\left(N(X)+i \varepsilon\|X\|^{2}\right)^{\frac{p+q+3}{2}}}, \\
& G_{p, q, \varepsilon} \nabla_{p, q}^{+}=i \varepsilon(p+q+1) \frac{\|X\|^{2}-X^{+} \bar{X}}{\left(N(X)+i \varepsilon\|X\|^{2}\right)^{\frac{p+q+3}{2}}} .
\end{aligned}
$$

By Lemma 12,

$$
d\left(G_{p, q, \varepsilon} \cdot D_{p, q} x \cdot f\right)=\left(G_{p, q, \varepsilon} \nabla_{p, q}^{+}\right) f d V_{p, q}+G_{p, q, \varepsilon}\left(\nabla_{p, q}^{+} f\right) d V_{p, q}=\left(G_{p, q, \varepsilon} \nabla_{p, q}^{+}\right) f d V_{p, q}
$$

since $f$ is $(p, q)$-left-monogenic. Then by Stokes' theorem we have

$$
\begin{aligned}
\int_{\partial U} G_{p, q, \varepsilon}(X) \cdot D_{p, q} x \cdot f(X) \\
\quad=\int_{U \backslash B_{r}} \frac{i \varepsilon(p+q+1)\left(\|X\|^{2}-X^{+} \bar{X}\right)}{\left(N(X)+i \varepsilon\|X\|^{2}\right)^{\frac{p+q+3}{2}}} f(X) d V_{p, q}+\int_{S_{r}} G_{p, q, \varepsilon}(X) \cdot D_{p, q} x \cdot f(X),
\end{aligned}
$$

where $B_{r}=\left\{X \in \mathbb{R}^{p+q+1} ;\|X\| \leq r\right\}$ is a ball of sufficiently small radius $r$ centered at the origin and $S_{r}$ is its boundary sphere.

Next, we establish (in order) analogues of Lemma 17, Lemma 18, and Lemma 20 of [L]. Recall that we have selected a branch of $z^{1 / 2}$ with values in the right half-plane, as discussed after Definition 3 .

Lemma 28. Fix a $\theta_{0} \in\left(0, \frac{\pi}{4}\right)$, and let $p, q$ be non-negative integers, then we have two distributions which send a test function $g(\theta)$ into the limits

$$
\lim _{\varepsilon \rightarrow 0^{+}} \int_{\frac{\pi}{4}-\theta_{0}}^{\frac{\pi}{4}+\theta_{0}} \frac{g(\theta) d \theta}{(\cos (2 \theta)+i \varepsilon)^{\frac{p+q+3}{2}}} \quad \text { and } \quad \lim _{\varepsilon \rightarrow 0^{-}} \int_{\frac{\pi}{4}-\theta_{0}}^{\frac{\pi}{4}+\theta_{0}} \frac{g(\theta) d \theta}{(\cos (2 \theta)+i \varepsilon)^{\frac{p+q+3}{2}}} .
$$

In other words, the above two limits of integrals define continuous linear functionals on the topological vector space of smooth function $\mathbb{1}^{1}$ on the interval $\left[\frac{\pi}{4}-\theta_{0}, \frac{\pi}{4}-\theta_{0}\right]$.

Proof. In the case of $p+q=1 \bmod 2$, this is a consequence of Lemma 17 of $[\mathrm{L}]$. Otherwise, we modify the proof to fit the fractional case. We have that $(p+q+3) / 2=n+1 / 2$ for some non-negative integer $n$, and we use induction on $n$. For the base case, $n=0$, we integrate by parts,

$$
\begin{gathered}
\int_{\frac{\pi}{4}-\theta_{0}}^{\frac{\pi}{4}+\theta_{0}} \frac{g(\theta) d \theta}{(\cos (2 \theta)+i \varepsilon)^{1 / 2}}=\int_{\frac{\pi}{4}-\theta_{0}}^{\frac{\pi}{4}+\theta_{0}} \frac{\sin (2 \theta)}{(\cos (2 \theta)+i \varepsilon)^{1 / 2}} \frac{g(\theta) d \theta}{\sin (2 \theta)} \\
=-\left.(\cos (2 \theta)+i \varepsilon)^{1 / 2} \frac{g(\theta)}{\sin (2 \theta)}\right|_{\pi / 4-\theta_{0}} ^{\pi / 4+\theta_{0}}+\int_{\frac{\pi}{4}-\theta_{0}}^{\frac{\pi}{4}+\theta_{0}}(\cos (2 \theta)+i \varepsilon)^{\frac{1}{2}} \frac{\mathrm{d}}{\mathrm{d} \theta}\left(\frac{g(\theta)}{\sin (2 \theta)}\right) d \theta .
\end{gathered}
$$

\footnotetext{
${ }^{1}$ Note that this vector space has the standard Frechét toplogy determined by the seminorms $\max _{\theta \in\left[\frac{\pi}{4}-\theta_{0}, \frac{\pi}{4}-\theta_{0}\right]} g^{(k)}(\theta)$, where $k=0,1,2, \ldots$
} 
As $(\cos (2 \theta)+i \varepsilon)^{1 / 2}$ is integrable for all values of $\varepsilon$, including $\varepsilon=0$, the limits as $\varepsilon \rightarrow 0^{ \pm}$ exist and depend continuously on $g(\theta)$. Now we consider the case of $n>0$, in which case we can integrate by parts,

$$
\begin{aligned}
\int_{\frac{\pi}{4}-\theta_{0}}^{\frac{\pi}{4}+\theta_{0}} & \frac{g(\theta) d \theta}{(\cos (2 \theta)+i \varepsilon)^{n+1 / 2}}=\int_{\frac{\pi}{4}-\theta_{0}}^{\frac{\pi}{4}+\theta_{0}} \frac{\sin (2 \theta)}{(\cos (2 \theta)+i \varepsilon)^{n+1 / 2}} \frac{g(\theta) d \theta}{\sin (2 \theta)} \\
& =\left.\frac{(\cos (2 \theta)+i \varepsilon)^{1 / 2-n}}{2 n-1} \frac{g(\theta)}{\sin (2 \theta)}\right|_{\pi / 4-\theta_{0}} ^{\pi / 4+\theta_{0}}-\int_{\frac{\pi}{4}-\theta_{0}}^{\frac{\pi}{4}+\theta_{0}} \frac{(2 n-1)^{-1}}{(\cos (2 \theta)+i \varepsilon)^{n-\frac{1}{2}}} \frac{\mathrm{d}}{\mathrm{d} \theta}\left(\frac{g(\theta)}{\sin (2 \theta)}\right) d \theta
\end{aligned}
$$

and the result follows by induction on $n$.

\section{Lemma 29.}

$$
\lim _{\varepsilon \rightarrow 0} \int_{U \backslash B_{r}} \frac{i \varepsilon(p+q+1)\left(\|X\|^{2}-X^{+} \bar{X}\right)}{\left(N(X)+i \varepsilon\|X\|^{2}\right)^{\frac{p+q+3}{2}}} f(X) d V_{p, q}=0 .
$$

Proof. We write the integral in the hybrid spherical coordinates (21) and integrate out the variables $r, \varphi_{1}, \ldots, \varphi_{n}, \psi_{p}, \ldots, \psi_{q-1}$. After we do this, we retain an integral of the form

$$
\varepsilon \int_{0}^{\frac{\pi}{2}} \frac{g(\theta) d \theta}{(\cos (2 \theta)+i \varepsilon)^{\frac{p+q+3}{2}}}
$$

for some function $g(\theta)$. Due to the transversality of the boundary $\partial U$ with respect to the null cone $\mathcal{N}_{p, q}=\left\{\theta=\frac{\pi}{4}\right\}$, the function $g(\theta)$ is smooth at least for $\theta$ lying in some interval $\left[\frac{\pi}{4}-\theta_{0}, \frac{\pi}{4}+\theta_{0}\right]$ with $\theta_{0} \in\left(0, \frac{\pi}{4}\right)$. We can apply Lemma 28 to $\int_{\frac{\pi}{4}-\theta_{0}}^{\frac{\pi}{4}+\theta_{0}} \ldots$, and the limit of the integral on the remainder of the interval exists, so the limit of (23) as $\varepsilon \rightarrow 0$ is zero.

We introduce a constant $C_{p, q}$ (it will be evaluated later in Lemma 33):

$$
C_{p, q}=\lim _{\varepsilon \rightarrow 0^{+}} \int_{0}^{\frac{\pi}{2}} \frac{\cos ^{p} \vartheta \sin ^{q-1} \vartheta}{(\cos (2 \vartheta)+i \varepsilon)^{\frac{p+q+1}{2}}} d \vartheta .
$$

Note that this limit exists by Lemma 28.

\section{Lemma 30.}

$$
\lim _{r \rightarrow 0^{+}}\left(\lim _{\varepsilon \rightarrow 0^{+}} \int_{S_{r}} \frac{r f(X) d S_{p, q}}{\left(N(X)+i \varepsilon r^{2}\right)^{\frac{p+q+1}{2}}}\right)=i^{q} \omega_{p} \omega_{q-1} C_{p, q} f(0) .
$$

Proof. By Lemma 25, we can split

$$
d S_{p, q}=i^{q} r^{p+q} \cos ^{p} \theta \sin ^{q-1} \theta d \theta d \Omega_{p, \varphi} d \Omega_{q-1, \psi}
$$

where

$$
d \Omega_{n, \alpha}=\rho^{-n} \operatorname{det}\left(S_{n, \alpha}\right) d \alpha_{1} d \alpha_{2} \ldots d \alpha_{n}
$$


represents the spherical volume element on the $n$-dimensional sphere. The factor of $i^{q}$ comes from the normalization of the Euclidean volume element on this space, (16). Note that the factors of $r$ in the numerator and denominator of

$$
\frac{r f(X) d S_{p, q}}{\left(N(X)+i \varepsilon r^{2}\right)^{\frac{p+q+1}{2}}}
$$

cancel. Let $F_{\varepsilon}(\theta)$ be the following antiderivative:

$$
F_{\varepsilon}(\theta)=\int_{0}^{\theta} \frac{\cos ^{p} \vartheta \sin ^{q-1} \vartheta}{(\cos (2 \vartheta)+i \varepsilon)^{\frac{p+q+1}{2}}} d \vartheta .
$$

Integrating by parts yields

$$
\begin{gathered}
\int_{S_{r}} \frac{r^{-p-q} f(X) d S_{p, q}}{(\cos (2 \theta)+i \varepsilon)^{\frac{p+q+1}{2}}}=i^{q} \int_{S_{q-1}} \int_{S_{p}} \int_{\theta=0}^{\theta=\pi / 2} \frac{f(X) \cos ^{p} \theta \sin ^{q-1} \theta d \theta d \Omega_{p, \varphi} d \Omega_{q-1, \psi}}{(\cos (2 \theta)+i \varepsilon)^{\frac{p+q+1}{2}}} \\
=\left.i^{q} \int_{S_{q-1}} \int_{S_{p}} f F_{\varepsilon}(\theta)\right|_{\theta=0} ^{\theta=\pi / 2} d \Omega_{p, \varphi} d \Omega_{q-1, \psi}-i^{q} \int_{S_{q-1}} \int_{S_{p}} F_{\varepsilon}(\theta) \frac{\partial f}{\partial \theta} d \theta d \Omega_{\varphi, p} d \Omega_{\psi, q-1} .
\end{gathered}
$$

By the chain rule, we have $\frac{\partial f}{\partial \theta}=\sum_{i} \frac{\partial f}{\partial x_{i}} \frac{\partial x_{i}}{\partial \theta}=r \cdot g(X)$, where $g(X)$ is a smooth function. Thus, the second term is bounded by a constant multiple of $r$ (by Lemma 28), and so in the limit of $r \rightarrow 0^{+}$it vanishes. In the first term, as $r \rightarrow 0^{+}, f$ approaches $f(0)$, and so we can carry it out, and integrate $d \Omega_{p, \varphi}$ and $d \Omega_{q-1, \psi}$ to be the respective volumes of unit spheres to obtain

$$
\begin{aligned}
\lim _{r \rightarrow 0^{+}}\left(\left.\lim _{\varepsilon \rightarrow 0^{+}} i^{q} \int_{S_{q-1}} \int_{S_{p}} f F_{\varepsilon}(\theta)\right|_{\theta=0} ^{\theta=\pi / 2} d \Omega_{p, \varphi} d \Omega_{q-1, \psi}\right) & \\
& =\left.f(0) \omega_{p} \omega_{q-1} \lim _{\varepsilon \rightarrow 0^{+}} F_{\varepsilon}(\theta)\right|_{\theta=0} ^{\theta=\pi / 2}=i^{q} \omega_{p} \omega_{q-1} C_{p, q} f(0) .
\end{aligned}
$$

The following integrals appear as certain cross terms.

Lemma 31. For each $0 \leq j \leq p$ and $1 \leq k \leq q$,

$$
\lim _{r \rightarrow 0^{+}}\left(\lim _{\varepsilon \rightarrow 0^{+}} \int_{S_{r}} \frac{x_{j} \tilde{x}_{p+k} f(X)}{\left(N(X)+i \varepsilon r^{2}\right)^{\frac{p+q+1}{2}}} \frac{d S_{p, q}}{r}\right)=0 .
$$

Proof. Note that, as in the proof of previous lemma, once we convert to the hybrid spherical coordinates (21), the factors of $r$ cancel. After conversion to these coordinates, the integrand depends on $\psi_{k}$ as follows. For $k=1, \ldots, q-1$, the integrand is of the form $\cos \psi_{k} \cdot h_{k}$, where $h_{k}$ is a function does not depend on $\psi_{k}$. And for $k=q$, it is of the form $\sin \psi_{q-1} \cdot h_{q}$. Additionally, we see from Lemma 25 that the Jacobian, as a function of $\psi_{k}$, is proportional to $\sin ^{q-1-k} \psi_{k}$ 
for $k=1, \ldots, q-1$. By absorbing the remaining factors of $x_{j}(\cos (2 \theta)+i \varepsilon)^{-\frac{p+q+1}{2}} d S_{p, q}$ into the $h_{k}$, so we still have $\frac{\partial h_{k}}{\partial \psi_{k}}=0$, for all $\varepsilon$, we can rewrite the integral as

$$
\begin{cases}\int_{S_{r}} \cos \psi_{k} \sin ^{q-1-k} \psi_{k} h_{k} f(X) d \theta d \varphi_{1} d \varphi_{2} \ldots d \varphi_{p} d \psi_{1} d \psi_{2} \ldots d \psi_{q-1} & \text { if } k=1, \ldots, q-2 \\ \int_{S_{r}} \cos \psi_{q-1} h_{q-1} f(X) d \theta d \varphi_{1} d \varphi_{2} \ldots d \varphi_{p} d \psi_{1} d \psi_{2} \ldots d \psi_{q-1} & \text { if } k=q-1, \\ \int_{S_{r}} \sin \psi_{q-1} h_{q} f(X) d \theta d \varphi_{1} d \varphi_{2} \ldots d \varphi_{p} d \psi_{1} d \psi_{2} \ldots d \psi_{q-1} & \text { if } k=q .\end{cases}
$$

For $1 \leq k \leq q-2$, when we integrate with respect to $\psi_{k}$, we have

$$
\begin{aligned}
\int_{\psi_{k}=0}^{\psi_{k}=\pi} & \cos \psi_{k} \sin ^{q-1-k} \psi_{k} h_{k} f(X) d \psi_{k} \\
& =\left.\frac{\sin ^{q-k} \psi_{k}}{q-k} h_{k} f(X)\right|_{\psi_{k}=0} ^{\psi_{k}=\pi}-\int_{0}^{\pi} \frac{\sin ^{q-k} \psi_{k}}{q-k} h_{k} \frac{\partial f}{\partial \psi_{k}} d \psi_{k}=-\int_{0}^{\pi} \frac{\sin ^{q-k} \psi_{k}}{q-k} h_{k} \frac{\partial f}{\partial \psi_{k}} d \psi_{k} .
\end{aligned}
$$

The partial derivative $\frac{\partial f}{\partial \psi_{k}}$ yields a factor of $r$ by the chain rule, as demonstrated in the proof of Lemma 30. Then we integrate out the remaining variables and let $\varepsilon \rightarrow 0^{+}$, the limit exists by Lemma 28. As $r \rightarrow 0^{+}$, the factor of $r$ ensures the limit is zero.

For $k=q-1$ and $k=q$, the bounds of integration are $0 \leq \psi_{q-1} \leq 2 \pi$, and the same argument works in this case as well.

Now we can prove an analogue of Lemma 20 of $[\mathrm{L}]$.

Lemma 32. Let $C_{p, q}$ be as in (24), then

$$
\lim _{r \rightarrow 0^{+}}\left(\lim _{\varepsilon \rightarrow 0^{+}} \int_{S_{r}} \frac{X^{+} D_{p, q} x f(X)}{\left(N(X)+i \varepsilon r^{2}\right)^{\frac{p+q+1}{2}}}\right)=i^{q} \omega_{p} \omega_{q-1} C_{p, q} f(0) .
$$

Proof. Using Corollary 15, we have

$$
\int_{S_{r}} \frac{X^{+} D_{p, q} x f(X)}{\left(N(X)+i \varepsilon r^{2}\right)^{\frac{p+q+1}{2}}}=\int_{S_{r}} \frac{X^{+} \bar{X} f(X)}{\left(N(X)+i \varepsilon r^{2}\right)^{\frac{p+q+1}{2}}} \frac{d S_{p, q}}{r} .
$$

We calculate, if $X=x_{0} e_{0}+\sum_{j=1}^{p} x_{j} e_{j}+\sum_{k=p+1}^{p+q} \tilde{x}_{k} \tilde{e}_{k}$,

$$
X^{+} \bar{X}=\|X\|^{2}-2 \sum_{j=0}^{p} \sum_{k=p+1}^{p+q} x_{j} \tilde{x}_{k} \tilde{e}_{k} e_{j} .
$$

We split the integral of (25) into two parts to be analyzed separately,

$$
\begin{aligned}
\int_{S_{r}} \frac{X^{+} D_{p, q} x f(X)}{\left(N(X)+i \varepsilon r^{2}\right)^{\frac{p+q+1}{2}}}=\int_{S_{r}} \frac{\|X\|^{2} f}{\left(N(X)+i \varepsilon r^{2}\right)^{\frac{p+q+1}{2}}} \frac{d S_{p, q}}{r} \\
\quad-2 \sum_{j=0}^{p} \sum_{k=p+1}^{p+q} \int_{S_{r}} \frac{x_{j} \tilde{x}_{k} \tilde{e}_{k} e_{j} f(X)}{\left(N(X)+i \varepsilon r^{2}\right)^{\frac{p+q+1}{2}}} \frac{d S_{p, q}}{r} .
\end{aligned}
$$

Applying Lemmas 30 and 31 yields the result. 
We have proved Theorem 23 up to a constant coefficient. Indeed, combining (22) with Lemmas 29 and 32, we obtain

$$
\lim _{\varepsilon \rightarrow 0^{+}} \int_{\partial U} G_{p, q, \varepsilon}\left(X-X_{0}\right) D_{p, q} x f(X)=i^{q} \omega_{p} \omega_{q-1} C_{p, q} f\left(X_{0}\right) \quad \text { if } X_{0} \in U .
$$

And if $X_{0} \notin \bar{U}$ the same argument shows that the limit of integrals is zero. Then Theorem 23 follows from Lemma 33 below.

\section{Lemma 33.}

$$
C_{p, q}=(-i)^{q} \frac{\omega_{p+q}}{\omega_{p} \omega_{q-1}}
$$

Proof. We prove this by applying both integral formulas to the constant function $f(X)=1$ on the sphere $S_{p, q}=\left\{X \in \mathbb{R}^{p+q+1}:\|X\|=1\right\}$. When we apply the first integral formula (Theorem 20), we obtain, for all $\varepsilon>0$ sufficiently close to zero,

$$
\int_{\left(h_{\varepsilon}\right)_{*}\left(S_{p, q}\right)} \frac{Z^{+} D_{p+q} z}{N(Z)^{\frac{p+q+1}{2}}}=\omega_{p+q} .
$$

We calculate the pullback of $D_{p+q} z$ and rewrite this as an integral over the sphere $S_{p, q}$. Note that $h_{\varepsilon}$ is a linear transformation and that

$$
\left(h_{\varepsilon}\right)^{*} d z_{j}= \begin{cases}(1+i \varepsilon) d z_{j} & \text { if } 0 \leq j \leq p \\ (1-i \varepsilon) d z_{j} & \text { if } p+1<\leq j \leq p+q .\end{cases}
$$

Using the expansion of $D_{p+q} z$ in the standard basis (14), we find:

$$
\begin{array}{r}
\left(h_{\varepsilon}\right)^{*} D_{p+q} z=\sum_{j=0}^{p}(-1)^{j} e_{j}(1+i \varepsilon)^{p}(1-i \varepsilon)^{q} d \hat{z}_{j}+\sum_{j=p+1}^{p+q}(-1)^{j} e_{j}(1+i \varepsilon)^{p+1}(1-i \varepsilon)^{q-1} d \hat{z}_{j} \\
=D_{p+q} z+\varepsilon \tilde{D}_{p+q, \varepsilon} z,
\end{array}
$$

where the form $\tilde{D}_{p+q, \varepsilon} z$ depends on $\varepsilon$ polynomially and does not depend on $Z$. Let $\tilde{D}_{p, q, \varepsilon} x$ be the restriction of $\tilde{D}_{p+q, \varepsilon} z$ to $\mathbb{R}^{p+q+1}$. Recall the conjugation (8). If $X \in \mathbb{R}^{p+q+1}$ and $Z=h_{\varepsilon}(X)$, then, using (20),

$$
\begin{gathered}
\left(h_{\varepsilon}\right)^{*} Z^{+}=(X+i \varepsilon \bar{X})^{+}=X^{+}+i \varepsilon \bar{X}^{+}, \\
\left(h_{\varepsilon}\right)^{*} N(Z)=\left(1-\varepsilon^{2}\right) N(X)+i \varepsilon\|X\|^{2}=\left(1-\varepsilon^{2}\right) N(X)+i \varepsilon .
\end{gathered}
$$

We have:

$$
\begin{aligned}
\int_{\left(h_{\varepsilon}\right)_{*}\left(S_{p, q}\right)} & \frac{Z^{+} D_{p+q} z}{N(Z)^{\frac{p+q+1}{2}}}=\int_{S_{p, q}} \frac{\left(X^{+}+i \varepsilon \bar{X}^{+}\right)\left(D_{p, q} x+\varepsilon \tilde{D}_{p, q, \varepsilon} x\right)}{\left(\left(1-\varepsilon^{2}\right) N(X)+i \varepsilon\right)^{\frac{p+q+1}{2}}} \\
= & \int_{S_{p, q}} \frac{X^{+} D_{p, q} x}{\left(\left(1-\varepsilon^{2}\right) N(X)+i \varepsilon\right)^{\frac{p+q+1}{2}}}+\int_{S_{p, q}} \frac{\varepsilon \bar{X}^{+} \tilde{D}_{p, q, \varepsilon} x+i \varepsilon \bar{X}^{+}\left(D_{p, q} x+\varepsilon \tilde{D}_{p, q, \varepsilon} x\right)}{\left(\left(1-\varepsilon^{2}\right) N(X)+i \varepsilon\right)^{\frac{p+q+1}{2}}} .
\end{aligned}
$$

As $\varepsilon \rightarrow 0^{+}$, the first integral approaches $i^{q} \omega_{p} \omega_{q-1} C_{p, q}$ by the already established formula $(26)$. And the second integral approaches zero because we can factor out $\varepsilon$ and proceed in the same manner as we proved Lemma 29 using the hybrid coordinates (21) and Lemma 28 . 


\section{References}

[BDS] F. Brackx, R. Delanghe, F. Sommen, Clifford Analysis, Pitman, London, 1982.

[CSSS] F. Colombo, I. Sabadini, F. Sommen, D. C. Struppa, Analysis of Dirac systems and computational algebra, Progress in Mathematical Physics, vol. 39, Birkhäuser Boston, 2004.

[DSS] R. Delanghe, F. Sommen, V. Souček, Clifford algebra and spinor-valued functions, Kluwer Academic Publishers Group, Dordrecht, 1992.

[FL] I. Frenkel, M. Libine, Split quaternionic analysis and the separation of the series for $S L(2, \mathbb{R})$ and $S L(2, \mathbb{C}) / S L(2, \mathbb{R})$, Advances in Math 228 (2011), 678-763; also arXiv:1009.2532.

[G] D. Garling, Clifford Algebras: An Introduction, London Math. Society Student Texts, Cambridge Univ. Press, 2011.

[GM] J. Gilbert, M. Murray, Clifford Algebras and Dirac Operators in Harmonic Analysis, Cambridge Univ. Press, Cambridge, UK, 1991.

[I] K. Imaeda, "A new formulation of classical electrodynamics", Nuovo Cimento B (11) 32 (1976), no. 1, 138-162.

[L] M. Libine, An invitation to split quaternionic analysis in I. Sabadini, F. Sommen (Eds.), Hypercomplex Analysis and its Applications, Birkhäuser, 2011, pp. 161-180; also arXiv:1009.2540.

[P] B. Prather, Split Octonionic Cauchy Integral Formula, Adv. Appl. Clifford Algebras 29 (2019), issue 5.

[RCW] G. Ren, L. Chen, H. Wang, Split-quaternionic Hermitian Clifford analysis, Complex Variables and Elliptic Equations 60 (2015), 333-353.

[R1] J. Ryan, Complexified Clifford analysis, Complex Variables, Theory and Appl. 1 (1982), 119-149.

[R2] J. Ryan, Cells of harmonicity and generalized Cauchy integral formulae, Proc. London Math. Soc. (3) 60 (1990), no. 2, 295-318.

[S] A. Sudbery, Quaternionic analysis, Math. Proc. Camb. Phil. Soc. 85 (1979), 199-225. 